\title{
Conditional Hyperbolic Quadrature Method of Moments for Kinetic Equations
}

\author{
Rodney O. Fox ${ }^{\mathrm{a}, \mathrm{b}, \mathrm{c}}$, Frédérique Laurent ${ }^{\mathrm{b}, \mathrm{c}}$, Aymeric Vié $^{\mathrm{b}, \mathrm{c}}$ \\ ${ }^{a}$ Department of Chemical and Biological Engineering, Iowa State University, 618 Bissell Road, Ames, IA 50011-1098, USA \\ ${ }^{b}$ Laboratoire EM2C, CNRS, CentraleSupélec, Université Paris-Saclay, Grande Voie des Vignes, 92295 Châtenay Malabry, \\ France \\ ${ }^{c}$ Fédération de Mathématiques de l'Ecole Centrale Paris, Grande Voie des Vignes, 92295 Châtenay Malabry, France
}

\begin{abstract}
The conditional quadrature method of moments (CQMOM) was introduced by Yuan and Fox [J. Comput. Phys. 230 (22), 8216-8246 (2011)] to reconstruct a velocity distribution function (VDF) from a finite set of its integer moments. The reconstructed VDF takes the form of a sum of weighted Dirac delta functions in velocity phase space, and provides a closure for the spatial flux term in the corresponding kinetic equation. The CQMOM closure for the flux leads to a weakly hyperbolic system of moment equations. In subsequent work [Chalons et al., Proceed. CTR Sum. Prog. 2010, 347-358 (2010)], the Dirac delta functions were replaced by Gaussian distributions, which make the moment system hyperbolic but at the added cost of dealing with continuous distributions. Here, a hyperbolic version of CQMOM is proposed that uses weighted Dirac delta functions. While the moment set employed for multi-Gaussian and conditional HyQMOM (CHyQMOM) are equivalent, the latter is able to access all of moment space whereas the former cannot (e.g. arbitrary values of the fourth-order velocity moment in 1-D phase space with two nodes). By making use of the properties of CHyQMOM in 2-D phase space, it is possible to control a symmetrical subset of the optimal moments [Fox, Indust. \& Engng. Chem. Res. 48 (21), 9686-9696 (2009)]. Furthermore, the moment sets for 2-D problems are smaller for CHyQMOM than in the original CQMOM thanks to a judicious choice of the velocity abscissas in phase space.
\end{abstract}

Keywords: kinetic equation, quadrature-based moment methods, conditional quadrature method of moments, hyperbolic moment closures

\section{Introduction}

The physics of inertial particles can be described by a velocity density function (VDF) satisfying a kinetic equation. Solving such a kinetic equation relies on either a sample of discrete numerical parcels through a Lagrangian Monte-Carlo method or on a moment approach resulting in a Eulerian system of conservation laws on velocity moments. For the latter, the main difficulty for particles with high Knudsen numbers where the VDF can be very far from equilibrium, is the closure of the free-transport term in the kinetic equation. One way to proceed is to use quadrature-based moment methods (QBMM) where the higher-order moments required for closure are evaluated from the lower-order transported moments using multi-dimensional quadrature $[1,2,3]$. In our previous work, we have developed the conditional quadrature method of moments (CQMOM) [4], leading to a well-behaved kinetic numerical scheme [5]. CQMOM has been shown to capture particle trajectory crossing (PTC) where the distribution in the exact kinetic equation remains at all times in the form of a sum of Dirac delta functions $[4,6,7]$. The moment system found with the CQMOM flux closure is weakly hyperbolic, leading to delta shocks when multiple PTC occur at the

Email addresses: rofox@iastate.edu (Rodney O. Fox), frederique.laurent@centralesupelec.fr (Frédérique Laurent), aymeric.vie@centralesupelec.fr (Aymeric Vié) 
same location. To achieve hyperbolicity, a multi-Gaussian QBMM closure was proposed [8, 9]. However, this closure cannot access all of moment space $[10,11,12]$ due to the form of Gaussian distribution (e.g. the 2node closure cannot represent fourth-order velocity moments larger than a Gaussian distribution). Moreover, working with a continuous VDF reconstruction loses the discrete velocity representation of CQMOM [13].

The purpose of this work is to introduce a hyperbolic QMOM reconstruction of the VDF (HyQMOM in one-dimensional (1-D) and CHyQMOM is two-dimensional (2-D) phase space) that circumvents the known shortcomings of the multi-Gaussian closure while retaining a hyperbolic moment system. In 1-D phase space, the moment set controlled by HyQMOM is exactly the same as with the multi-Gaussian closure, namely integer moments up to order $2 N$ where $N$ is the number of nodes. Here we analyze the HyQMOM for $N=2$ and 3 , extend it to 2 -D phase space using a modified version of CQMOM, and apply it to the solution of a kinetic equation. The remainder of the work is organized as follows. In $\S 2$ the HyQMOM is described. In $\S 3$, we provide an in-depth description of the application to 1-D kinetic equations and the mathematical properties of 2- and 3-node HyQMOM. In $\S 4$, we extend HyQMOM to a 2-D phase space using CHyQMOM. In $\S 5$ we describe the application of $\mathrm{CHyQMOM}$ to 2-D kinetic equations. Example applications are provided in $\S 6$. Finally, conclusions are drawn in $\S 7$. Mathematical details on moment methods and extension of CHyQMOM to higher-order moments are provided in the appendices.

\section{HyQMOM}

First, the moments and the central moments are defined, and the realizability conditions for the moments are given. Then, HyQMOM is defined and the particular cases with three and five moments are described in detail.

\subsection{Moments, central moments, and realizability}

Consider a VDF $f(u)$ defined for $u \in \mathbb{R}$. Let us assume that the moments of $f$ defined by

$$
M_{k}:=\int_{\mathbb{R}} f(u) u^{k} \mathrm{~d} u \quad \text { for } k \in\{0,1, \ldots, p\}
$$

are finite. Let us also denote by $\mathbf{M}_{p}=\left(M_{0}, M_{1}, \ldots, M_{p}\right)^{t}$ the corresponding moment vector.

In what follows, the use of central moments will allow to simplify the computations. For $M_{0}>0$, they are defined by

$$
C_{k}:=\frac{1}{M_{0}} \int_{\mathbb{R}} f(u)(u-\bar{u})^{k} \mathrm{~d} v \quad \text { for } k \in\{0,1, \ldots, p\}
$$

where $\bar{u}=M_{1} / M_{0}$. By definition, $C_{0}=1$ and $C_{1}=0$. The next central moment $C_{2} \geq 0$ is the velocity variance. For any $k \geq 2$, the central moment $C_{k}$ depends uniquely on the moment set $\mathbf{M}_{k}$ through the relation

$$
C_{k}=\sum_{i=0}^{k}\left(\begin{array}{c}
k \\
i
\end{array}\right)\left(-\frac{M_{1}}{M_{0}}\right)^{k-i} \frac{M_{i}}{M_{0}} .
$$

And inversely, for $k \geq 2, M_{k}$ depends uniquely on the vector $\mathbf{C}_{k}=\left(M_{0}, \bar{u}, C_{2}, \ldots, C_{k}\right)^{t}$ through the relation

$$
M_{k}=M_{0}\left[\sum_{i=2}^{k}\left(\begin{array}{l}
k \\
i
\end{array}\right) \bar{u}^{k-i} C_{i}+\bar{u}^{k}\right] .
$$

For a positive measure $\mathrm{d} \mu(u)=f(u) \mathrm{d} u$, the moment vector $\mathbf{M}_{2 N-2}$ is said to be realizable $\left(\mathbf{M}_{2 N-2}\right.$ is in the moment space). Moreover, if the support of $\mathrm{d} \mu(u)$ contains more than $N$ points, then $\mathbf{M}_{2 N-2}$ is said to be strictly realizable $\left(\mathbf{M}_{2 N-2}\right.$ is in the interior of the moment space). The realizability of a finite set of moments can be checked using Hankel matrix determinants as described in Appendix A. The corresponding constraints can be more easily written on the central moments. In particular, up to fourth order the central moments are strictly realizable if and only if $C_{2}>0$ and $C_{4}>C_{2}^{2}+C_{3}^{2} / C_{2}$. Hereinafter we will assume that the moment set under consideration is realizable. 


\subsection{Definition of $H y Q M O M$}

From a moment vector $\mathbf{M}_{2 N-2}$, with $N \geq 2$, HyQMOM provides a discrete approximation $f^{a}$ defined such that

$$
M_{k}=M_{k}^{a}:=\int_{\mathbb{R}} f^{a}(u) u^{k} \mathrm{~d} u \quad \text { for } k \in\{0,1, \ldots, 2 N-1\},
$$

where the additional moment $M_{2 N-1}$ is a function of $\mathbf{M}_{2 N-2}$. It can be equivalently written with the corresponding central moments, and then using the vector $\mathbf{C}_{2 N-2}=\left(M_{0}, \bar{u}, C_{2}, \ldots, C_{2 N-2}\right)^{t}$. Thus the discrete approximation $f^{a}$ is defined such that

$$
C_{k}=C_{k}^{a}:=\frac{1}{M_{0}} \int_{\mathbb{R}} f^{a}(u)(u-\bar{u})^{k} \mathrm{~d} u \quad \text { for } k \in\{0,1, \ldots, \max (1,2 N-2)\},
$$

with $C_{0}=1$ and $C_{1}=0$ and the additional central moment $C_{2 N-1}$ is a function of $\left(C_{2}, C_{3}, \ldots, C_{2 N-2}\right)$.

The discrete approximation $f^{a}$ has the form

$$
f^{a}(u)=\sum_{\alpha=1}^{N} w_{\alpha} \delta_{v_{\alpha}}(u)
$$

where $\delta_{v_{\alpha}}(u)$ is the Dirac delta function centered at $v_{\alpha}$, and the $N$ non-negative weights $w_{\alpha}$ and the $N$ velocity abscissas $v_{\alpha}$ are determined from the first $2 N-1$ integer moments of $f^{a}$ by (5). Using the central moments and denoting $w_{\alpha}=M_{0} \rho_{\alpha}$ and $v_{\alpha}=u_{\alpha}+\bar{u}$, it is equivalent to

$$
C_{k}=\sum_{\alpha=1}^{N} \rho_{\alpha} u_{\alpha}^{k} \quad \text { for } k \in\{0,1, \ldots, 2 N-1\} .
$$

In comparison to QMOM, HyQMOM fixes the central moment $C_{2 N-1}$, instead of computing it from moments up to $M_{2 N-1}$, such that the 1-D moment system in $\S 3.1$ is hyperbolic. The algorithm for computing $f^{a}$ from the moment set $\left\{C_{0}, \ldots, C_{2 N-1}\right\}$ using (8) is exactly the same as with QMOM [4, 14]. As shown below, for $N=2$ and 3 the weights $\rho_{\alpha}$ and velocity abscissas $u_{\alpha}$ can be found analytically. Note that due to the form of (7), we effectively constraint the even-order moment $C_{2 N}$. (See Appendix A for details.)

The odd-order central moments have no such constraints. We therefore choose $C_{2 N-1}$ such that two conditions hold: (1) the moment system found from the 1-D kinetic equation is hyperbolic (i.e. the $2 N-1$ eigenvalues $\lambda_{n}$ are real and distinct) and (2) one eigenvalue corresponds to the mean velocity $\bar{u}$. In this work, we focus on the moment systems with $N \in\{2,3\}$. The open problem of choosing $C_{2 N-1}$ for $4 \leq N$ is briefly discussed in Appendix A. Finally, it is important to note that the choice of $C_{2 N-1}$ does not rely on reconstructing the VDF (i.e., it does not use any properties of Gaussian quadrature). In this sense, it is truly a nonlinear moment closure for $C_{2 N-1}$ given $\left\{C_{2}, C_{3}, \ldots, C_{2 N-2}\right\}$.

\subsection{Two-node HyQMOM}

The function $f^{a}$ has exact moments $M_{i}^{a}$ of orders $i=0,1,2$ given by (8) with $N=2$. The four unknowns $\rho_{1}, \rho_{2}, u_{1}, u_{2}$ are found by solving the nonlinear system $C_{i}=C_{i}^{a}, i=0, \ldots, 3$ :

$$
\begin{aligned}
1 & =\rho_{1}+\rho_{2}, \\
0 & =\rho_{1} u_{1}+\rho_{2} u_{2}, \\
C_{2} & =\rho_{1} u_{1}^{2}+\rho_{2} u_{2}^{2}, \\
C_{3} & =\rho_{1} u_{1}^{3}+\rho_{2} u_{2}^{3},
\end{aligned}
$$

with the closure $C_{3}=0$. Note that if $0<C_{2}$, then the abscissas can be rescaled by $\sqrt{C_{2}}$ :

$$
\begin{aligned}
& 1=\rho_{1}+\rho_{2}, \\
& 0=\rho_{1} u_{1}^{\prime}+\rho_{2} u_{2}^{\prime}, \\
& 1=\rho_{1}\left(u_{1}^{\prime}\right)^{2}+\rho_{2}\left(u_{2}^{\prime}\right)^{2}, \\
& 0=\rho_{1}\left(u_{1}^{\prime}\right)^{3}+\rho_{2}\left(u_{2}^{\prime}\right)^{3},
\end{aligned}
$$


where $u^{\prime}=u / \sqrt{C_{2}}$. Thus, (9) has no free parameters. It remains to prove that this system is well posed in the following proposition.

Proposition 1 (2-node HyQMOM). For moment set $\mathbf{M}=\left(M_{0}, M_{1}, M_{2}\right)^{t}$ such that $M_{0}>0$, define the central moment

$$
C_{2}=\frac{M_{0} M_{2}-M_{1}^{2}}{M_{0}^{2}}
$$

and set $C_{3}=0$. System (9) has a unique solution for any $\mathbf{M}$ in the interior $\Omega$ of the moment space, which is given by

$$
\Omega=\left\{\mathbf{M}, M_{0}>0 \text { and } C_{2}>0\right\} .
$$

Setting $\mathbf{U}=\left(w_{1}, w_{2}, w_{1} v_{1}, w_{2} v_{2}\right)^{t}$, where $w_{\alpha}=M_{0} \rho_{\alpha}$ and $v_{\alpha}=u_{\alpha}+\bar{u}$, the function $\mathbf{U}=\mathbf{U}(\mathbf{M})$ is a bijection from $\Omega$ to $\Omega_{U}=\left\{\mathbf{U}, w_{1}=w_{2}>0\right.$ and $\left.w_{1} v_{1} \neq w_{2} v_{2}\right\}$.

Proof. In the case $C_{2}>0$, using the second equation, the last two equations in (10) yield

$$
\begin{aligned}
\rho_{1} u_{1}^{\prime}\left(u_{1}^{\prime}-u_{2}^{\prime}\right) & =1, \\
u_{1}^{\prime}+u_{2}^{\prime} & =0 .
\end{aligned}
$$

Using the first equation in (10), these equations then yield

$$
\begin{array}{r}
\rho_{1}=\rho_{2}=\frac{1}{2}, \\
u_{1}^{\prime}=-u_{2}^{\prime}=1 .
\end{array}
$$

Moreover, this leads to $w_{1}=w_{2}=\frac{M_{0}}{2}>0$ and $v_{1}=\bar{u}+\sqrt{C_{2}}$ and $v_{2}=\bar{u}-\sqrt{C_{2}}$, in such a way that $v_{1} \neq v_{2}$. This concludes the proof.

If $M_{0}=0$, then $\mathbf{M}$ is realizable only if $M_{1}=M_{2}=0$. When $C_{2}=0,2$-node HyQMOM reduces to 1-node QMOM.

\subsection{Three-node HyQMOM}

The function $f^{a}$ has exact central moments $C_{i}^{a}$ of orders $i=0, \ldots, 4$ given by (8) with $N=3$. The six unknowns $\rho_{1}, \rho_{2}, \rho_{3}, u_{1}, u_{2}, u_{3}$ are found by solving the nonlinear system

$$
\begin{aligned}
1 & =\rho_{1}+\rho_{2}+\rho_{3}, \\
0 & =\rho_{1} u_{1}+\rho_{2} u_{2}+\rho_{3} u_{3}, \\
C_{2} & =\rho_{1} u_{1}^{2}+\rho_{2} u_{2}^{2}+\rho_{3} u_{3}^{2}, \\
C_{3} & =\rho_{1} u_{1}^{3}+\rho_{2} u_{2}^{3}+\rho_{3} u_{3}^{3}, \\
C_{4} & =\rho_{1} u_{1}^{4}+\rho_{2} u_{2}^{4}+\rho_{3} u_{3}^{4}, \\
C_{5} & =\rho_{1} u_{1}^{5}+\rho_{2} u_{2}^{5}+\rho_{3} u_{3}^{5},
\end{aligned}
$$

with the closure $C_{5}=\frac{C_{3}}{C_{2}^{2}}\left(2 C_{2} C_{4}-C_{3}^{2}\right)$ so that $u_{2}=0$. Note again that the abscissas can be rescaled by $\sqrt{C_{2}}$ and thus (13) depends only on the two dimensionless moments $q:=C_{3} / C_{2}^{3 / 2}$ and $\eta:=C_{4} / C_{2}^{2}$. It remains to prove that this system is well posed in the following proposition.

Proposition 2 (3-node HyQMOM). For moment set $\mathbf{M}=\left(M_{0}, M_{1}, M_{2}, M_{3}, M_{4}\right)^{t}$ such that $M_{0}>0$, define the central moments

$$
C_{2}=\frac{M_{0} M_{2}-M_{1}^{2}}{M_{0}^{2}}, \quad C_{3}=\frac{M_{0}^{2} M_{3}-3 M_{0} M_{1} M_{2}+2 M_{1}^{3}}{M_{0}^{3}},
$$




$$
C_{4}=\frac{M_{0}^{3} M_{4}-4 M_{0}^{2} M_{1} M_{3}+6 M_{0} M_{1}^{2} M_{2}-3 M_{1}^{4}}{M_{0}^{4}}
$$

and set $C_{5}=\frac{C_{3}}{C_{2}^{2}}\left(2 C_{2} C_{4}-C_{3}^{2}\right)$. System (13) has a unique solution for any $\mathbf{M}$ in the space $\Omega$ defined by

$$
\Omega=\left\{\mathbf{M}, M_{0}>0, C_{2}>0 \text { and } C_{4} \geq C_{2}^{2}+\frac{C_{3}^{2}}{C_{2}}\right\} .
$$

Setting $\mathbf{U}=\left(w_{1}, w_{2}, w_{3}, w_{1} v_{1}, w_{2} v_{2}, w_{3} v_{3}\right)^{t}$, where $w_{\alpha}=M_{0} \rho_{\alpha}$ and $v_{\alpha}=u_{\alpha}+\bar{u}$, the function $\mathbf{U}=\mathbf{U}(\mathbf{M})$ is a bijection from $\Omega$ to $\Omega_{U}=\left\{\mathbf{U}, w_{1}>0, w_{2} \geq 0, w_{3}>0\right.$ and $\left.\frac{w_{2} v_{2}}{w_{2}}=\frac{w_{1} v_{1}+w_{3} v_{3}}{w_{1}+w_{3}}\right\}$.

Proof. Solving (13) with $u_{2}=0$ is equivalent to solving the following nonlinear system in $\left(\rho_{1}^{*}, \rho_{2}, \rho_{3}^{*}, u_{1}, u_{3}\right)$ :

$$
\begin{aligned}
\rho_{2} & =1-\rho_{1}^{*} / u_{1}-\rho_{3}^{*} / u_{3}, \\
\rho_{1}^{*}+\rho_{3}^{*} & =0, \\
\rho_{1}^{*} u_{1}+\rho_{3}^{*} u_{3} & =C_{2}, \\
\rho_{1}^{*} u_{1}^{2}+\rho_{3}^{*} u_{3}^{2} & =C_{3}, \\
\rho_{1}^{*} u_{1}^{3}+\rho_{3}^{*} u_{3}^{3} & =C_{4}, \\
C_{5} & =\rho_{1}^{*} u_{1}^{4}+\rho_{3}^{*} u_{3}^{4} .
\end{aligned}
$$

In the case $C_{2}>0$, the last five equations yield

$$
\begin{aligned}
\rho_{3}^{*} & =-\rho_{1}^{*}, \\
\rho_{1}^{*}\left(u_{1}-u_{3}\right) & =C_{2}, \\
u_{1}+u_{3} & =C_{3} / C_{2}=C_{2}^{1 / 2} q, \\
u_{1}^{2}+u_{1} u_{3}+u_{3}^{2} & =C_{4} / C_{2}=C_{2} \eta, \\
C_{5} & =C_{3}\left(u_{1}^{2}+u_{3}^{2}\right) .
\end{aligned}
$$

The last three equations then yield

$$
\begin{aligned}
& u_{1}=C_{2}^{1 / 2} \frac{1}{2}\left(q-\sqrt{4 \eta-3 q^{2}}\right), \\
& u_{3}=C_{2}^{1 / 2} \frac{1}{2}\left(q+\sqrt{4 \eta-3 q^{2}}\right),
\end{aligned}
$$

and

$$
C_{5}=C_{2}^{5 / 2} q\left(2 \eta-q^{2}\right)
$$

which give real-valued abscissas with $u_{1}<0$ and $u_{3}>0$ when $4 \eta \geq 3 q^{2}$. The corresponding weights are

$$
\rho_{1}=\frac{-C_{2}^{1 / 2}}{u_{1} \sqrt{4 \eta-3 q^{2}}}, \quad \rho_{2}=1+\frac{C_{2}}{u_{1} u_{3}}, \quad \rho_{3}=\frac{C_{2}^{1 / 2}}{u_{3} \sqrt{4 \eta-3 q^{2}}} .
$$

Using only the moments, the latter can be written as

$$
\rho_{1}=\frac{1}{2\left(\eta-q^{2}\right)}\left(1+\frac{q}{\sqrt{4 \eta-3 q^{2}}}\right), \quad \rho_{2}=1-\frac{1}{\eta-q^{2}}, \quad \rho_{3}=\frac{1}{2\left(\eta-q^{2}\right)}\left(1-\frac{q}{\sqrt{4 \eta-3 q^{2}}}\right) .
$$

The weights are non-negative if $\eta \geq 1+q^{2}$, which is the realizability condition for M. Moreover, with this condition, the abscissas found from (16) are always real-valued. Finally, $\rho_{1} u_{1}+\rho_{3} u_{3}=0$ is equivalent to $\bar{u}=\frac{w_{1} v_{1}+w_{3} v_{3}}{w_{1}+w_{3}}$. This concludes the proof. 
If $M_{0}=0$, then $\mathbf{M}$ is realizable only if $M_{1}=M_{2}=M_{3}=M_{4}=0$. If $C_{2}=0$, then $\mathbf{M}$ is realizable only if $C_{3}=C_{4}=0$, in which case $\rho_{1}=\rho_{3}=0$. When $C_{2} C_{4}=C_{2}^{3}+C_{3}^{2}$, the weight $\rho_{2}=0$ and, hence, the 3 -node HyQMOM reduces to 2-node QMOM at the boundary of moment space. Finally, let us remark that for the 3-node HyQMOM, thanks to (4), the fifth-order moment is given in terms of the central moments by

$$
M_{5}=M_{0}\left[\frac{C_{3}}{C_{2}^{2}}\left(2 C_{2} C_{4}-C_{3}^{2}\right)+5 \bar{u} C_{4}+10 \bar{u}^{2} C_{3}+10 \bar{u}^{3} C_{2}+\bar{u}^{5}\right] .
$$

\section{Application of HyQMOM to 1-D kinetic equations}

We first introduce HyQMOM for the $\operatorname{VDF} f(t, x, u)$ in 1-D phase/real space for the kinetic equation:

$$
\partial_{t} f+u \partial_{x} f+\partial_{u}(\mathcal{A} f)=0, \quad t>0, x \in \mathbb{R}, u \in \mathbb{R}
$$

with initial condition $f(0, x, u)=f_{0}(x, u)$. The acceleration $\mathcal{A}$ is a real-valued function of $u$. The exact solution for free transport (when $\mathcal{A}=0$ ) is given by $f(t, x, u)=f(0, x-u t, u)=f_{0}(x-u t, u)$. In this work, we seek an approximation of $f(t, x, u)$ in the form of HyQMOM with weights $\rho_{\alpha}(t, x)>0$, velocity abscissas $u_{\alpha}(t, x)$ for $\alpha \in(1, \ldots, N)$ and $N=3$. These weights and abscissas are determined from the 1-D moment transport equations.

\subsection{1-D moment transport equations}

Defining the $i^{\text {th }}$-order moment:

$$
M_{i}(t, x)=\int_{\mathbb{R}} f(t, x, u) u^{i} \mathrm{~d} u, \quad i=0, \ldots, K ; \quad K \in \mathbb{N}
$$

the associated governing equations are easily obtained from (21) after multiplication by $u^{i}$ and integration over $u$ :

$$
\partial_{t} M_{i}+\partial_{x} M_{i+1}=\overline{\mathcal{A}}_{i}, i \geq 0
$$

where the (unclosed $)^{1}$ moment acceleration term is

$$
\overline{\mathcal{A}}_{i}=-\int_{\mathbb{R}} i \mathcal{A}(u) f(t, x, u) u^{i-1} \mathrm{~d} u .
$$

For simplicity, we will focus our attention on the 5-moment model and its abstract form:

$$
\begin{aligned}
\partial_{t} M_{0}+\partial_{x} M_{1} & =0 \\
\partial_{t} M_{1}+\partial_{x} M_{2} & =\overline{\mathcal{A}}_{1}, \\
\partial_{t} M_{2}+\partial_{x} M_{3} & =\overline{\mathcal{A}}_{2}, \quad \Longrightarrow \quad \partial_{t} \mathbf{M}+\partial_{x} \mathbf{F}(\mathbf{M})=\overline{\mathbf{A}} \\
\partial_{t} M_{3}+\partial_{x} M_{4} & =\overline{\mathcal{A}}_{3}, \\
\partial_{t} M_{4}+\partial_{x} \bar{M}_{5} & =\overline{\mathcal{A}}_{4} .
\end{aligned}
$$

with $\mathbf{M}=\left(M_{0}, \ldots, M_{4}\right)^{t}, \mathbf{F}(\mathbf{M})=\left(M_{1}, \ldots, M_{4}, \bar{M}_{5}\right)^{t}$ and $\overline{\mathbf{A}}=\left(0, \overline{\mathcal{A}}_{1}, \ldots, \overline{\mathcal{A}}_{4}\right)^{t}$. This model is closed provided that $\bar{M}_{5}$ and $\overline{\mathbf{A}}$ are defined as functions of $\mathbf{M}$. Here we propose to define these functions using 3-node HyQMOM. Note that unlike the 5-moment closures in [9, 10, 12], 3-node HyQMOM in (23) is well defined over the entire (realizable) moment space $\mathbf{M}$. It is also noteworthy that $\bar{M}_{5}$ can be expressed as an analytical function of $\mathbf{M}$. Thus, the flux Jacobian matrix $\partial \mathbf{F} / \partial \mathbf{M}$, whose eigenvalues determine the hyperbolicity of (23), can be expressed in terms of $\mathbf{M}$.

\footnotetext{
${ }^{1}$ The acceleration terms will be closed if $\mathcal{A}$ is affine: $\mathcal{A}(t, x, u)=-a(t, x) u+b(t, x)$, in which case the moment acceleration term can be written as $\overline{\mathcal{A}}_{k}=k\left(a M_{k}-b M_{k-1}\right)$. In gas-particle flows, this limit corresponds to Stokes drag in a stationary fluid.
} 


\subsection{Mathematical properties of moment system with HyQMOM}

The following theorem addresses an important mathematical property of system (23).

Theorem 1 (Hyperbolicity). Assuming that the vector $\mathbf{M}=\left(M_{0}, M_{1}, M_{2}, M_{3}, M_{4}\right)^{t}$ lives in the space $\Omega$ defined in Proposition 2, system (23) with the 3-node HyQMOM closure for $\bar{M}_{5}$ given by (20) is hyperbolic.

Proof. First, let us write a system of equations on the vector $\mathbf{C}=\left(M_{0}, \bar{u}, C_{2}, C_{3}, C_{4}\right)^{t}$ equivalent to (23) for regular solutions. It can be written

$$
\frac{D \mathbf{M}}{D \mathbf{C}} \partial_{t} \mathbf{C}+\frac{D \mathbf{F}(\mathbf{M})}{D \mathbf{C}} \partial_{x} \mathbf{C}=\overline{\mathbf{A}},
$$

in such a way that

$$
\partial_{t} \mathbf{C}+J \partial_{x} \mathbf{C}=\left(\frac{D \mathbf{M}}{D \mathbf{C}}\right)^{-1} \overline{\mathbf{A}}, \quad J=\left(\frac{D \mathbf{M}}{D \mathbf{C}}\right)^{-1} \frac{D \mathbf{F}(\mathbf{M})}{D \mathbf{C}} .
$$

The Jacobian matrix $J$ of this system is given by

$$
J=\left[\begin{array}{ccccc}
\bar{u} & M_{0} & 0 & 0 & 0 \\
\frac{C_{2}}{M_{0}} & \bar{u} & 1 & 0 & 0 \\
\frac{C_{3}}{M_{0}} & 2 C_{2} & \bar{u} & 1 & 0 \\
\frac{-3 C_{2}^{2}+C_{4}}{M_{0}} & 3 C_{3} & -3 C_{2} & \bar{u} & 1 \\
-\frac{C_{3}}{M_{0} C_{2}^{2}}\left(4 C_{2}^{3}-2 C_{4} C_{2}+C_{3}^{2}\right) & 4 C_{4} & -\frac{2 C_{3}}{C_{2}^{3}}\left(2 C_{2}^{3}+C_{4} C_{2}-C_{3}^{2}\right) & -\frac{3 C_{3}^{2}-2 C_{2} C_{4}}{C_{2}^{2}} & \bar{u}+\frac{2 C_{3}}{C_{2}}
\end{array}\right] .
$$

The corresponding characteristic polynomial $P$ is then such that

$$
C_{2}^{-5 / 2} P\left(\bar{u}+\sqrt{C_{2}} Y\right)=-Y^{5}+2 q Y^{4}+\left(2 \eta-3 q^{2}\right) Y^{3}-2 q\left(\eta-q^{2}\right) Y^{2}-\left(\eta-q^{2}\right) Y
$$

with $q=C_{3} / C_{2}^{3 / 2}$ and $\eta=C_{4} / C_{2}^{2}$. This polynomial function $P$ then admits five roots:

$$
\bar{u}, \quad \bar{u}+\sqrt{C_{2}} \frac{1}{2}\left(q \pm \sqrt{4 \eta-3 q^{2} \pm 4 \sqrt{\left(\eta-q^{2}\right)\left(\eta-q^{2}-1\right)}}\right) .
$$

Thanks to the realizability constraint $\eta>1+q^{2}$, it is easy to show that all roots are distinct and real-valued. This concludes the proof.

If $\eta=1+q^{2}$, the moments are on the boundary of moment space and system (23) is weakly hyperbolic [6]. Using the same procedure as in Theorem 1, it can be shown that the moment system resulting from 2-node HyQMOM is hyperbolic when $C_{2}>0$.

\subsection{Kinetic-based flux}

A finite-volume scheme corresponding to the transport part of (23) can be written [9]:

$$
\mathrm{d}_{t} \mathbf{M}_{j}(t)=-\frac{1}{\Delta x_{j}}\left[\mathbf{F}_{j+1 / 2}(t)-\mathbf{F}_{j-1 / 2}(t)\right]
$$

where $\mathbf{M}_{j}=\left(M_{0}, \ldots, M_{4}\right)^{t}$ is an approximation of the mean value of the moment vector on the $j^{\text {th }}$ cell ]$x_{j-1 / 2}, x_{j+1 / 2}$ [ of size $\Delta x_{j}$. In our numerical implementation to solve (23), the spatial fluxes $\mathbf{F}_{j+1 / 2}=$ $\left(F_{0, j+1 / 2}, \ldots, F_{4, j+1 / 2}\right)^{t}$ are computed using a kinetic-based definition [15]:

$$
F_{k, j+1 / 2}(t)=\int_{0}^{\infty} v^{k+1} f_{j}\left(t, x_{j+1 / 2}, v\right) \mathrm{d} v+\int_{-\infty}^{0} v^{k+1} f_{j+1}\left(t, x_{j+1 / 2}, v\right) \mathrm{d} v, \quad k=0, \ldots, 4,
$$

where $f_{j}$ is a reconstruction of the VDF in the $j^{\text {th }}$ cell. A decomposition into positive and negative directions is then used to define the flux function as proposed in $[5,16]$. The numerical representation of the flux 
function is a critical point in moment transport methods $[1,2,3,7,9,17]$ because only realizable moment sets can be successfully inverted.

For the reconstruction of the VDF, we can choose either the 3-node HyQMOM reconstruction in (7), or a reconstruction based on the eigenvalues of the moment system. For the former, we have

$$
F_{k, j+1 / 2}(t)=\sum_{\alpha=1}^{3}\left[\left.M_{0} \rho_{\alpha} \max \left(0, u_{\alpha}+\bar{u}\right)^{k+1}\right|_{j}+\left.M_{0} \rho_{\alpha} \min \left(0, u_{\alpha}+\bar{u}\right)^{k+1}\right|_{j+1}\right], \quad k=0, \ldots, 4,
$$

where the subscript $j$ indicates the reconstruction in cell $j$. Alternatively, we can close (28) using

$$
F_{k, j+1 / 2}(t)=\sum_{\alpha=0}^{4}\left[\left.M_{0} w_{\alpha} \max \left(0, \lambda_{\alpha}\right)^{k+1}\right|_{j}+\left.M_{0} w_{\alpha} \min \left(0, \lambda_{\alpha}\right)^{k+1}\right|_{j+1}\right], \quad k=0, \ldots, 4,
$$

where $\lambda_{\alpha}$ are the five eigenvalues in (26) with $\lambda_{0}=\bar{u}$. This definition of the numerical flux will only be realizable when the weights $w_{\alpha}$ are non-negative [7].

The weights $w_{\alpha}$ in (30) are found by solving the moment problem:

$$
M_{0}\left[\begin{array}{ccccc}
1 & 1 & 1 & 1 & 1 \\
\lambda_{0} & \lambda_{1} & \lambda_{2} & \lambda_{3} & \lambda_{4} \\
\lambda_{0}^{2} & \lambda_{1}^{2} & \lambda_{2}^{2} & \lambda_{3}^{2} & \lambda_{4}^{2} \\
\lambda_{0}^{3} & \lambda_{1}^{3} & \lambda_{2}^{3} & \lambda_{3}^{3} & \lambda_{4}^{3} \\
\lambda_{0}^{4} & \lambda_{1}^{4} & \lambda_{2}^{4} & \lambda_{3}^{4} & \lambda_{4}^{4}
\end{array}\right]\left[\begin{array}{c}
w_{0} \\
w_{1} \\
w_{2} \\
w_{3} \\
w_{4}
\end{array}\right]=\left[\begin{array}{c}
M_{0} \\
M_{1} \\
M_{2} \\
M_{3} \\
M_{4}
\end{array}\right]
$$

By replacing the moments $M_{k}$ in (31) with their equivalent expressions written in terms of the central moments, we find that $w_{0}=0$ and

$$
\left[\begin{array}{cccc}
\varphi_{1} & \varphi_{2} & \varphi_{3} & \varphi_{4} \\
\varphi_{1}^{2} & \varphi_{2}^{2} & \varphi_{3}^{2} & \varphi_{4}^{2} \\
\varphi_{1}^{3} & \varphi_{2}^{3} & \varphi_{3}^{3} & \varphi_{4}^{3} \\
\varphi_{1}^{4} & \varphi_{2}^{4} & \varphi_{3}^{4} & \varphi_{4}^{4}
\end{array}\right]\left[\begin{array}{l}
w_{1} \\
w_{2} \\
w_{3} \\
w_{4}
\end{array}\right]=\left[\begin{array}{l}
0 \\
1 \\
q \\
\eta
\end{array}\right]
$$

where $\varphi_{\alpha}=\left(\lambda_{\alpha}-\bar{u}\right) / \sqrt{C_{2}}$ are the normalized eigenvalues. Remarkably, the linear system in (32) yields non-negative weights for all realizable values of $\eta$. For the degenerate case where $\eta=1+q^{2}$, the three distinct eigenvalues are used in (30), which is then equivalent to (29). Finally, for the degenerate case where $C_{2}=0$, the single eigenvalue $\bar{u}$ is used.

To design a first-order scheme, the decomposition in (30) is sufficient as it corresponds to an upwind scheme at the kinetic level. For a high-order scheme [7], the spatial fluxes can be found, for example, from (30) by employing a high-order spatial reconstruction for $M_{0} w_{\alpha}$ and a first-order reconstruction for the abscissas $\lambda_{\alpha}$. In summary, with the numerical fluxes in (30), the moments are advanced in time as follows:

Step 1. Given moments $\mathbf{M}_{j}^{n}$, compute $\bar{u}, C_{2}, q, \eta, \lambda_{\alpha}$, and $w_{\alpha}$ in each grid cell.

Step 2. Compute kinetic-based fluxes from (30).

Step 3. Advance the moments in time to find $\mathbf{M}_{j}^{n+1}$ using (27) with a CFL number based on the largest $\left|\lambda_{\alpha}\right|$ in the computational domain.

The source terms on the right-hand side of (23) are treated using operator splitting [9].

\section{Extension of HyQMOM to 2-D phase space}

Consider a 2-D phase space with $\operatorname{VDF} f(\mathrm{v})$ for $\mathrm{v}=(u, v)^{t}$ and define the bivariate moments

$$
M_{i, j}:=\int_{\mathbb{R}^{2}} f(\mathrm{v}) u^{i} v^{j} \mathrm{dv}, i, j=0, \ldots, K ; K \in \mathbb{N} .
$$


If $M_{0,0}>0$, the bivariate central moments are defined by

$$
C_{i, j}:=\frac{1}{M_{0,0}} \int_{\mathbb{R}^{2}} f(\mathrm{v})(u-\bar{u})^{i}(v-\bar{v})^{j} \mathrm{dv}, i, j=0, \ldots, K ; K \in \mathbb{N}
$$

where $\bar{u}=M_{1,0} / M_{0,0}$ and $\bar{v}=M_{0,1} / M_{0,0}$. Assuming that these moments are realizable, which in principle can be checked employing methods developed for multivariate distributions [18, 19, 20, 21, 22, 23], in the following we propose a bivariate extension of HyQMOM using ideas from CQMOM [4].

\subsection{Moments needed for 2-D CHyQMOM}

The moment-inversion algorithm described below uses the following ten velocity moments:

$$
\mathbf{M}=\left[\begin{array}{lllll}
M_{0,0} & M_{0,1} & M_{0,2} & M_{0,3} & M_{0,4} \\
M_{1,0} & M_{1,1} & & & \\
M_{2,0} & & & & \\
M_{3,0} & & & & \\
M_{4,0} & & & &
\end{array}\right],
$$

which is a symmetrical subset of the optimal moments [24]. The formulas developed in this section for 2-D, 9-node CHyQMOM can be extended to third-order moments in a relatively straightforward manner (see Appendix B) by including $M_{1,2}$ and $M_{2,1}$ in $\mathrm{M}$. When $0<M_{0,0}$, working with (35) is equivalent to working with $M_{0,0}, \bar{u}, \bar{v}$ and the central moments

$$
\mathbf{C}=\left[\begin{array}{ccccc}
1 & 0 & C_{0,2} & C_{0,3} & C_{0,4} \\
0 & C_{1,1} & & & \\
C_{2,0} & & & & \\
C_{3,0} & & & & \\
C_{4,0} & & & &
\end{array}\right] .
$$

Thus, in the following, we will express the 2-D reconstruction algorithm in terms of (36).

\subsection{Definition of 2-D CHyQMOM}

For clarity, we limit our discussion here to 9-node quadrature in 2-D phase space. Nevertheless, the same methodology can be used to develop the formulas for more nodes. For the 9-node quadrature, we define an approximate bivariate VDF by

$$
f^{a}(\mathrm{v}):=M_{0,0} \sum_{\alpha=1}^{3} \rho_{\alpha} \delta_{\bar{u}+u_{\alpha}}(u) \sum_{\beta=1}^{3} \rho_{\alpha \beta} \delta_{\bar{v}+\bar{v}_{\alpha}+v_{\alpha \beta}}(v),
$$

and the parameters $\left\{\rho_{1}, \rho_{2}, \rho_{3}, u_{1}, u_{3}\right\}\left(u_{2}=0\right)$ are determined using the 3-node HyQMOM algorithm in $\S 2.4$ from the central moments $\left\{1,0, C_{2,0}, C_{3,0}, C_{4,0}\right\}$. In (37), the three conditional velocities $\bar{v}_{\alpha}$ and the nine abscissas $v_{\alpha \beta}$ are found from the central moments $\left\{C_{1,1}, 0, C_{0,2}, C_{0,3}, C_{0,4}\right\}$ as described next. The total number of nodes in 2-D phase space is thus nine for non-degenerate cases.

In the first step, ${ }^{2}$ the two coefficients $a_{0}$ and $a_{1}$ in the conditional velocity $\bar{v}_{\alpha}=a_{0}+a_{1} u_{\alpha}$ appearing in (37) are defined by forcing $\bar{v}_{\alpha}$ to satisfy the following two conditions:

$$
\sum_{\alpha=1}^{3} \rho_{\alpha} \bar{v}_{\alpha}=C_{0,1}=0, \quad \sum_{\alpha=1}^{3} \rho_{\alpha} u_{\alpha} \bar{v}_{\alpha}=C_{1,1} .
$$

\footnotetext{
${ }^{2}$ Unlike in the original algorithm for CQMOM [4], the modified CQMOM used here fits the conditional moments to loworder polynomials where the coefficients are the unknowns. Thus, the number of coefficients (and the order of the polynomial) depends on the number of moments that can be matched in the reconstruction.
} 
This yields $a_{0}=0$ and $a_{1}=C_{1,1} / C_{2,0}$, and thus the conditional velocities are defined by

$$
\bar{v}_{\alpha}=\frac{C_{1,1}}{C_{2,0}} u_{\alpha}
$$

Note that $\bar{v}_{2}=0$ because $u_{2}=0$.

Next, we can note that the central moments found from (37) are

$$
C_{i, j}=\sum_{\alpha=1}^{3} \rho_{\alpha} u_{\alpha}^{i} \sum_{\beta=1}^{3} \rho_{\alpha \beta}\left(\bar{v}_{\alpha}+v_{\alpha \beta}\right)^{j} .
$$

A binomial expansion then leads to

$$
C_{i, j}=\sum_{j_{1}=0}^{j}\left(\begin{array}{c}
j \\
j_{1}
\end{array}\right) \sum_{\alpha=1}^{3} \rho_{\alpha} u_{\alpha}^{i} \bar{v}_{\alpha}^{j-j_{1}} C_{j_{1} \mid \alpha}
$$

where the conditional central moments are defined by

$$
C_{j \mid \alpha}:=\sum_{\beta=1}^{3} \rho_{\alpha \beta} v_{\alpha \beta}^{j} .
$$

It follows immediately from (41) that $C_{0 \mid \alpha}=1$ (i.e., this is the only solution that yields the known $C_{i, 0}$ from (41)). By setting $C_{2,1}=C_{1,1} C_{3,0} / C_{2,0}$, from (41) with the three central moments $\left\{C_{0,1}, C_{1,1}, C_{2,1}\right\}$, we find $C_{1 \mid \alpha}=0 .^{3}$ Note that this solution is unique as long as $0<\rho_{\alpha}$ and $u_{1} \neq u_{3}$, which is true for non-degenerate (i.e., strictly realizable) moments. The form of (41) then leads to the following moment-inversion algorithm based on CQMOM to find the remaining parameters $\rho_{\alpha \beta}$ and $v_{\alpha \beta}$.

\subsection{Moment-inversion algorithm for 2-D, 9-node CHyQMOM}

The univariate central moments $C_{i, 0}$ are used with the algorithm in $\S 2.4$ for $1-\mathrm{D}, 3$-node HyQMOM to find $\left\{\rho_{1}, \rho_{2}, \rho_{3}, u_{1}, u_{3}\right\}$. There are three possible cases: (1) a non-degenerate case with $u_{1} \neq u_{3}$ and $\rho_{2}>0$, (2) a degenerate case with $\rho_{2}=1,(3)$ a degenerate case with $\rho_{2}=0$. Case (2) occurs when $C_{2,0}=0$. Case

(3) occurs when the univariate moments are on the boundary of moment space, namely, when $C_{2,0}>0$ and $C_{2,0} C_{4,0}=C_{2,0}^{3}+C_{3,0}^{2}$.

\subsubsection{Case (2)}

For this case, we define a 2-D, 3-node CHyQMOM by

$$
f^{a}(\mathrm{v}):=M_{0,0} \delta_{\bar{u}}(u) \sum_{\beta=1}^{3} \rho_{\beta} \delta_{\bar{v}+v_{\beta}}(v) .
$$

The algorithm in $\S 2.4$ for 3-node HyQMOM is employed with the central moment set $\left\{1,0, C_{0,2}, C_{0,3}, C_{0,4}\right\}$ to find the parameters $\left\{\rho_{1}, \rho_{2}, \rho_{3}, v_{1}, v_{3}\right\}\left(v_{2}=0\right)$.

\footnotetext{
${ }^{3}$ Conversely, if $C_{1 \mid \alpha}=0$, then $C_{2,1}=C_{1,1} C_{3,0} / C_{2,0}$. Because $C_{2,1}$ is not in the transported moment set, we are free to close it in a consistent manner. See Appendix B for the reconstruction algorithm when $C_{1,2}$ and $C_{2,1}$ are known. The closure for $C_{2,1}$ is found be setting $a_{2}=0$ in the linear system (B.3).
} 


\subsubsection{Cases (1) and (3)}

The five parameters $\left\{\rho_{\alpha 1}, \rho_{\alpha 2}, \rho_{\alpha 3}, v_{\alpha 1}, v_{\alpha 2},\right\}$ are determined from the five conditional central moments $\left\{1,0, C_{2 \mid \alpha}, C_{3 \mid \alpha}, C_{4 \mid \alpha}\right\}$ using the 3 -node HyQMOM in $\S 2.4$. Thus, we begin by finding the two coefficients $b_{0}$ and $b_{1}$ appearing in the conditional variance defined by

$$
C_{2 \mid \alpha}=C_{0,2}\left(b_{0}+b_{1} \frac{u_{\alpha}}{C_{2,0}^{1 / 2}}\right) .
$$

Using (41) with $\left\{C_{0,2}, C_{1,2}\right\}$ where $C_{1,2}=C_{1,1} C_{0,3} / C_{0,2},{ }^{4}$ this yields

$$
\begin{aligned}
\sum_{\alpha=1}^{3} \rho_{\alpha} C_{2 \mid \alpha} & =C_{0,2} b_{0}=C_{0,2}-\frac{C_{1,1}^{2}}{C_{2,0}} \text { or } b_{0}=1-\varrho^{2}, \\
\sum_{\alpha=1}^{3} \rho_{\alpha} u_{\alpha} C_{2 \mid \alpha} & =b_{1} C_{2,0}^{1 / 2} C_{0,2}=C_{1,1} \frac{C_{0,3}}{C_{0,2}}-\frac{C_{1,1}^{2}}{C_{2,0}^{2}} C_{3,0} \quad \text { or } \quad b_{1}=\varrho\left(q_{2}-\varrho q_{1}\right)
\end{aligned}
$$

where $\varrho=C_{1,1} / \sqrt{C_{2,0} C_{0,2}}$ is the correlation coefficient, and the skewness coefficients are defined as $q_{1}=$ $C_{3,0} / C_{2,0}^{3 / 2}$ and $q_{2}=C_{0,3} / C_{0,2}^{3 / 2}$. If one of the conditional variances found from (44) is null, then $b_{1}$ is limited such that all conditional variances are non-negative. Note that when the limiter is applied, the value of $C_{1,2}$ will not equal $C_{1,1} C_{0,3} / C_{0,2}$; however, none of the moments in $\mathbf{C}$ will be affected.

In the final step, conditional moments $C_{3 \mid \alpha}$ and $C_{4 \mid \alpha}$ are found from the two central moments $\left\{C_{0,3}, C_{0,4}\right\}$ by assuming that the conditional moments depend on $\alpha$ through $C_{2 \mid \alpha}$, namely, $C_{3 \mid \alpha}=q^{\star} C_{2 \mid \alpha}^{3 / 2}$ and $C_{4 \mid \alpha}=$ $\eta^{\star} C_{2 \mid \alpha}^{2}$. From (41), this yields the following relations for $q^{\star}$ and $\eta^{\star}$ :

$$
q^{\star}=\left[\sum_{\alpha=1}^{3} \rho_{\alpha}\left(C_{2 \mid \alpha}^{\dagger}\right)^{3 / 2}\right]^{-1}\left[2 \varrho^{3} q_{1}+\left(1-3 \varrho^{2}\right) q_{2}\right]
$$

where $C_{2 \mid \alpha}^{\dagger}=C_{2 \mid \alpha} / C_{0,2}$, and

$$
\eta^{\star}=\left[\sum_{\alpha=1}^{3} \rho_{\alpha}\left(C_{2 \mid \alpha}^{\dagger}\right)^{2}\right]^{-1}\left[\eta_{2}-\varrho^{4} \eta_{1}-6 \varrho\left[\varrho\left(1-\varrho^{2}\right)+\left(q_{2}-\varrho q_{1}\right) q_{1}\right]-4 \varrho q^{\star} \sum_{\alpha=1}^{3} \rho_{\alpha} u_{\alpha}^{\dagger}\left(C_{2 \mid \alpha}^{\dagger}\right)^{3 / 2}\right]
$$

where $u_{\alpha}^{\dagger}=u_{\alpha} / C_{2,0}^{1 / 2}, \eta_{1}=C_{4,0} / C_{2,0}^{2}$ and $\eta_{2}=C_{0,4} / C_{0,2}^{2}$.

In the limit of perfect correlation, $|\varrho|=1, q_{2}=\varrho q_{1}$ and $\eta_{2}=\varrho^{2} \eta_{1}$, and thus $q^{\star}=0$ and $\eta^{\star}=0$. For uncorrelated variables, $\varrho=0, q^{\star}=q_{2}$ and $\eta^{\star}=\eta_{2}$. Otherwise, the realizability of $C_{4 \mid \alpha}$ requires that $\eta^{\star} \geq 1+\left(q^{\star}\right)^{2}$. If this condition is not met, then $q^{\star}$ and $\eta^{\star}$ are projected to the realizability curve $\eta^{\star}=1+\left(q^{\star}\right)^{2}$ along the direction of the Gaussian moments (i.e., $q^{\star}=0$ and $\eta^{\star}=3$ ). Three-node HyQMOM can then be applied for each $\alpha$ to find the remaining parameters $\left\{\rho_{\alpha 1}, \rho_{\alpha 2}, \rho_{\alpha 3}, v_{\alpha 1}, v_{\alpha 2},\right\}$. When this projection step is applied, the VDF reconstruction does not reproduce the moments $C_{0,3}$ and $C_{0,4}$. Moreover, $\rho_{\alpha 2}=0$ for all $\alpha$ in this case.

\subsection{Other remarks}

The reconstruction in (37) in not unique for this moment set, which is not surprising since a general 2-D, 9-node quadrature has 27 degrees of freedom [24]. For example, transposing the moments $M_{i, j} \Rightarrow M_{j, i}$ will generally lead to a different set of reconstruction parameters. The difference between the two reconstructions

\footnotetext{
${ }^{4}$ The value of $C_{1,2}$ is needed to compute $b_{1}$ in (44). Because it is not in the transported moment set, $C_{1,2}$ is closed analogously to $C_{2,1}$.
} 
will lead to different closures for the moments not included in $\mathbf{M}$. As shown in the numerical examples in $\S 6$, the advantage of using CHyQMOM over CQMOM [4], second-order closures [8], and the multi-Gaussian closure [9] for approximating solutions to kinetic equations is that the system will be hyperbolic, while at the same time allowing for particle-trajectory crossing $[1,2]$ for any realizable moment vector $\mathbf{M}$.

As discussed in $\$ 3.3$, for the spatial fluxes a reconstruction based on the eigenvalues can be employed. Using the flux-based reconstruction in the $x$ direction, the approximate VDF is

$$
f^{a}(\mathrm{v}):=M_{0,0} \sum_{\alpha=1}^{4} w_{\alpha} \delta_{\lambda_{\alpha}}(u) \sum_{\beta=1}^{3} \rho_{\alpha \beta} \delta_{\bar{v}+\bar{v}_{\alpha}+v_{\alpha \beta}}(v)
$$

where the eigenvalues are $\lambda_{\alpha}=\bar{u}+\sqrt{C_{2,0}} \varphi_{\alpha}$ and the weights $w_{\alpha}$ are found by solving (32). The formulas for 2-D reconstruction given above were derived using the notation in (37), but equivalent formulas can be found for (48) by substituting $w_{\alpha}$ for $\rho_{\alpha}$ and $\varphi_{\alpha}$ for $u_{\alpha} / C_{2,0}^{1 / 2}$. The latter are needed for computing the kinetic-based fluxes in $§ 5.3$.

\section{Application of 9-node CHyQMOM to kinetic equations}

Consider a 2-D velocity phase space with $\operatorname{VDF} f(t, \mathrm{x}, \mathrm{v})$ for $\mathrm{x}=(x, y)^{t}$ and $\mathrm{v}=(u, v)^{t}$ that satisfies the kinetic equation

$$
\partial_{t} f+\mathrm{v} \cdot \partial_{\mathrm{x}} f+\partial_{\mathrm{v}} \cdot(\mathbf{A} f)=0, \quad t>0, \mathrm{x} \in \mathbb{R}^{2}, \mathrm{v} \in \mathbb{R}^{2},
$$

with initial condition $f(0, \mathrm{x}, \mathrm{v})=f_{0}(\mathrm{x}, \mathrm{v})$. The acceleration $\mathbf{A}=\left(\mathcal{A}_{x}, \mathcal{A}_{y}\right)^{t}$ is a real-valued function of v. With a 2-D velocity phase space, we approximate the solution to $f$ using CHyQMOM for the bivariate moments. In this work, we will consider only the minimal CHyQMOM in $\S 4$ that uses nine nodes in the $2-\mathrm{D}$ velocity phase space. Nonetheless, the extension to more than nine nodes would be analogous to the algorithm presented here.

\subsection{2-D moment transport equations}

Defining the bivariate moments

$$
M_{i, j}(t, \mathrm{x})=\int_{\mathbb{R}^{2}} f(t, \mathrm{x}, \mathrm{v}) u^{i} v^{j} \mathrm{dv}, i, j=0, \ldots, K ; K \in \mathbb{N}
$$

the associated governing equations are easily obtained from (49):

$$
\partial_{t} M_{i, j}+\partial_{x} M_{i+1, j}+\partial_{y} M_{i, j+1}=\overline{\mathcal{A}}_{i, j}, \quad i, j \geq 0 ;
$$

where the (unclosed $)^{5}$ moment acceleration term is defined by

$$
\overline{\mathcal{A}}_{i, j}=-\int_{\mathbb{R}^{2}} i \mathcal{A}_{x}(\mathrm{v}) f(t, \mathrm{x}, \mathrm{v}) u^{i-1} v^{j} \mathrm{dv}-\int_{\mathbb{R}^{2}} j \mathcal{A}_{y}(\mathrm{v}) f(t, \mathrm{x}, \mathrm{v}) u^{i} v^{j-1} \mathrm{dv} .
$$

\footnotetext{
${ }^{5}$ The acceleration terms will be closed if $\mathbf{A}$ is a linear function of the form $(a u, a v)^{t}$, in which case the moment acceleration term can be written as $\overline{\mathcal{A}}_{i, j}=-a(i+j) M_{i, j}$. In gas-particle flows, this limit corresponds to Stokes drag in a stationary fluid.
} 
We will consider in this work the ten moments in (35):

$$
\begin{aligned}
\partial_{t} M_{0,0}+\partial_{x} M_{1,0}+\partial_{y} M_{0,1} & =0, \\
\partial_{t} M_{1,0}+\partial_{x} M_{2,0}+\partial_{y} M_{1,1} & =\overline{\mathcal{A}}_{1,0}, \\
\partial_{t} M_{0,1}+\partial_{x} M_{1,1}+\partial_{y} M_{0,2} & =\overline{\mathcal{A}}_{0,1}, \\
\partial_{t} M_{2,0}+\partial_{x} M_{3,0}+\partial_{y} \bar{M}_{2,1} & =\overline{\mathcal{A}}_{2,0}, \\
\partial_{t} M_{1,1}+\partial_{x} \bar{M}_{2,1}+\partial_{y} \bar{M}_{1,2} & =\overline{\mathcal{A}}_{1,1}, \\
\partial_{t} M_{0,2}+\partial_{x} \bar{M}_{1,2}+\partial_{y} M_{0,3} & =\overline{\mathcal{A}}_{0,2}, \\
\partial_{t} M_{3,0}+\partial_{x} M_{4,0}+\partial_{y} \bar{M}_{3,1} & =\overline{\mathcal{A}}_{3,0}, \\
\partial_{t} M_{0,3}+\partial_{x} \bar{M}_{1,3}+\partial_{y} M_{0,4} & =\overline{\mathcal{A}}_{0,3}, \\
\partial_{t} M_{4,0}+\partial_{x} \bar{M}_{5,0}+\partial_{y} \bar{M}_{4,1} & =\overline{\mathcal{A}}_{4,0}, \\
\partial_{t} M_{0,4}+\partial_{x} \bar{M}_{1,4}+\partial_{y} \bar{M}_{0,5} & =\overline{\mathcal{A}}_{0,4},
\end{aligned}
$$

which requires a closure for the two third-order moments $\bar{M}_{2,1}, \bar{M}_{1,2}$, the two fourth-order moments $\bar{M}_{3,1}$, $\bar{M}_{1,3}$, the four fifth-order moments $\bar{M}_{5,0}, \bar{M}_{4,1}, \bar{M}_{1,4}, \bar{M}_{0,5}$, and the acceleration terms. We propose to define these closures by reconstructing $f$ with 9-node CHyQMOM in (37) and (48). If unclosed, the acceleration term $\overline{\mathbf{A}}$ can be evaluated using $f^{a}$. In our numerical examples, Stokes drag is used so that $\overline{\mathbf{A}}$ is closed in terms of the transported moments, and operator splitting is used for the fluxes and the acceleration.

\subsection{Mathematical properties of 2-D moment system with 9-node CHyQMOM}

Because we employ a dimensional splitting to solve (51), let us consider the transport part of the system in the $x$-direction for the moments used in the CHyQMOM reconstruction in (37):

$$
\partial_{t} \mathbf{M}+\partial_{x} \mathbf{F}_{x}(\mathbf{M})=\mathbf{0}
$$

with $\mathbf{M}=\left(M_{0,0}, M_{1,0}, M_{2,0}, M_{3,0}, M_{4,0}, M_{0,1}, M_{1,1}, M_{0,2}, M_{0,3}, M_{0,4}\right)^{t}$ and

$$
\mathbf{F}_{x}(\mathbf{M})=\left(M_{1,0}, M_{2,0}, M_{3,0}, M_{4,0}, \bar{M}_{5,0}, M_{1,1}, \bar{M}_{2,1}, \bar{M}_{1,2}, \bar{M}_{1,3}, \bar{M}_{1,4}\right)^{t}
$$

where, using the flux-based quadrature in (48),

$$
\begin{gathered}
\bar{M}_{5,0}=M_{0,0} \sum_{\alpha=1}^{4} w_{\alpha} \lambda_{\alpha}^{5} \\
\bar{M}_{2,1}=M_{0,0} \sum_{\alpha=1}^{4} w_{\alpha} \lambda_{\alpha}^{2}\left(\bar{v}+\bar{v}_{\alpha}\right), \\
\bar{M}_{1,2}=M_{0,0} \sum_{\alpha=1}^{4} w_{\alpha} \lambda_{\alpha}\left[\left(\bar{v}+\bar{v}_{\alpha}\right)^{2}+C_{2 \mid \alpha}\right] \\
\bar{M}_{1,3}=M_{0,0} \sum_{\alpha=1}^{4} w_{\alpha} \lambda_{\alpha}\left[\left(\bar{v}+\bar{v}_{\alpha}\right)^{3}+3\left(\bar{v}+\bar{v}_{\alpha}\right) C_{2 \mid \alpha}+C_{3 \mid \alpha}\right] \\
\bar{M}_{1,4}=M_{0,0} \sum_{\alpha=1}^{4} w_{\alpha} \lambda_{\alpha}\left[\left(\bar{v}+\bar{v}_{\alpha}\right)^{4}+6\left(\bar{v}+\bar{v}_{\alpha}\right)^{2} C_{2 \mid \alpha}+4\left(\bar{v}+\bar{v}_{\alpha}\right) C_{3 \mid \alpha}+C_{4 \mid \alpha}\right] .
\end{gathered}
$$

Here $\bar{v}_{\alpha}=\left(C_{1,1} / C_{2,0}^{1 / 2}\right) \varphi_{\alpha}$, and the conditional moments $C_{j \mid \alpha}$ are defined using $\varphi_{\alpha}$. The right-hand side of (53) can therefore be expressed in terms of the known central moments, $\bar{u}, \bar{v}$ and $M_{0,0}$. This observation leads to the following theorem. 
Theorem 2 (Hyperbolicity). Assuming that the moment-inversion algorithm for 2-D, 9-node CHyQMOM for the vector $\mathbf{M}$ is non-degenerate, system (52) with this closure is hyperbolic for small $|\varrho|$.

Proof. The additional fluxes for the 2-D problem can be written as

$$
\begin{gathered}
\bar{M}_{2,1}=\bar{v} M_{2,0}+M_{0,0} C_{1,1}\left(2 \bar{u}+\frac{C_{3,0}}{C_{2,0}}\right) \\
\bar{M}_{1,2}=\bar{u} M_{0,2}+M_{0,0} C_{1,1}\left(2 \bar{v}+\frac{C_{0,3}}{C_{0,2}}\right) \\
\bar{M}_{1,3}=\bar{u} M_{0,3}+3 \bar{v} M_{0,0} C_{1,1}\left(\bar{v}+\frac{C_{0,3}}{C_{0,2}}\right)+M_{0,0} \bar{C}_{1,3}, \\
\bar{M}_{1,4}=\bar{u} M_{0,4}+2 \bar{v}^{2} M_{0,0} C_{1,1}\left(2 \bar{v}+3 \frac{C_{0,3}}{C_{0,2}}\right)+M_{0,0}\left(4 \bar{v} \bar{C}_{1,3}+\bar{C}_{1,4}\right)
\end{gathered}
$$

where, using the flux-based quadrature in (48),

$$
\begin{gathered}
\bar{C}_{1,3}=\sqrt{C_{2,0}} \sum_{\alpha=1}^{4} w_{\alpha} \varphi_{\alpha}\left(\bar{v}_{\alpha}^{3}+3 \bar{v}_{\alpha} C_{2 \mid \alpha}+C_{3 \mid \alpha}\right), \\
\bar{C}_{1,4}=\sqrt{C_{2,0}} \sum_{\alpha=1}^{4} w_{\alpha} \varphi_{\alpha}\left(\bar{v}_{\alpha}^{4}+6 \bar{v}_{\alpha}^{2} C_{2 \mid \alpha}+4 \bar{v}_{\alpha} C_{3 \mid \alpha}+C_{4 \mid \alpha}\right),
\end{gathered}
$$

and the eigenvalues $\varphi_{\alpha}$ are found from the eigenvalues $\lambda_{\alpha}$ of the 1-D system as shown below. These eigenvalues have the property $\sum_{\alpha=1}^{4} w_{\alpha} \varphi_{\alpha}^{k}=C_{k, 0} / C_{2,0}^{k / 2}$ for $k=0,1, \ldots, 5$ where $C_{0,0}:=1$.

The Jacobian matrix $J_{2 D}$ of the fluxes is block triangular:

$$
J_{2 D}=\left[\begin{array}{cccc}
J & 0 & 0 & 0 \\
X_{1} & A_{1} & 0 & 0 \\
X_{2} & B_{1} & A_{2} & 0 \\
X_{3} & C & B_{2} & \lambda_{10}
\end{array}\right]
$$

where $J$ is the $5 \times 5$ Jacobian matrix given in Theorem 1, corresponding to the 1 -D system. $X_{1}$ and $X_{2}$ are $2 \times 5$ matrices and $X_{3}$ is a $1 \times 5$ matrix. $B_{1}$ is a $2 \times 2$ matrix. $C$ and $B_{2}$ are $1 \times 2$ matrices. $A_{1}$ is a $2 \times 2$ matrix given by

$$
A_{1}=\left[\begin{array}{ll}
0 & 1 \\
\nu & \xi
\end{array}\right]
$$

with

$$
(\nu, \xi)=\frac{D \bar{M}_{2,1}}{D\left(M_{0,1}, M_{1,1}\right)}=\left(C_{2,0}-\bar{u}^{2}-\bar{u} \frac{C_{3,0}}{C_{2,0}}, 2 \bar{u}+\frac{C_{3,0}}{C_{2,0}}\right) .
$$

$A_{2}$ is a $2 \times 2$ matrix given by

$$
A_{2}=\left[\begin{array}{ll}
a_{11} & a_{12} \\
a_{21} & a_{22}
\end{array}\right]
$$

with

$$
\begin{gathered}
\left(a_{11}, a_{12}\right)=\frac{D \bar{M}_{1,2}}{D\left(M_{0,2}, M_{0,3}\right)}=\left(\bar{u}-\frac{C_{1,2}}{C_{0,2}}-3 \bar{v} \frac{C_{1,1}}{C_{0,2}}, \frac{C_{1,1}}{C_{0,2}}\right), \\
a_{21}=\frac{\partial \bar{M}_{1,3}}{\partial M_{0,2}}=-3 \bar{v} \frac{C_{1,2}}{C_{0,2}}-9 \bar{v}^{2} \frac{C_{1,1}}{C_{0,2}}+M_{0,0} \frac{\partial \bar{C}_{1,3}}{\partial M_{0,2}} \\
a_{22}=\frac{\partial \bar{M}_{1,3}}{\partial M_{0,3}}=\bar{u}+3 \bar{v} \frac{C_{1,1}}{C_{0,2}}+M_{0,0} \frac{\partial \bar{C}_{1,3}}{\partial M_{0,3}}
\end{gathered}
$$

where $C_{1,2}=C_{1,1} C_{0,3} / C_{0,2}$. 
Using the dimensionless variables $\bar{u}^{\dagger}=\bar{u} / C_{2,0}^{1 / 2}, \bar{v}^{\dagger}=\bar{v} / C_{0,2}^{1 / 2}, \varrho=C_{1,1} /\left(C_{2,0} C_{0,2}\right)^{1 / 2}, q_{1}=C_{3,0} / C_{2,0}^{3 / 2}$, $q_{2}=C_{0,3} / C_{0,2}^{3 / 2}, \eta_{1}=C_{4,0} / C_{2,0}^{2}$, and $\eta_{2}=C_{0,4} / C_{0,2}^{2}$, the partial derivatives can be expressed in dimensionless form as

$$
\begin{aligned}
& \left(\frac{\partial \bar{C}_{1,3}}{\partial M_{0,2}}\right)^{\dagger}=\sum_{\alpha=1}^{4} w_{\alpha} \varphi_{\alpha}\left[3\left(\varrho \varphi_{\alpha}+\frac{1}{2} q^{\star}\left(C_{2 \mid \alpha}^{\dagger}\right)^{1 / 2}\right)\left(\frac{\partial C_{2 \mid \alpha}}{\partial M_{0,2}}\right)^{\dagger}+\left(C_{2 \mid \alpha}^{\dagger}\right)^{3 / 2}\left(\frac{\partial q^{\star}}{\partial M_{0,2}}\right)^{\dagger}\right], \\
& \left(\frac{\partial \bar{C}_{1,3}}{\partial M_{0,3}}\right)^{\dagger}=\sum_{\alpha=1}^{4} w_{\alpha} \varphi_{\alpha}\left[3\left(\varrho \varphi_{\alpha}+\frac{1}{2} q^{\star}\left(C_{2 \mid \alpha}^{\dagger}\right)^{1 / 2}\right)\left(\frac{\partial C_{2 \mid \alpha}}{\partial M_{0,3}}\right)^{\dagger}+\left(C_{2 \mid \alpha}^{\dagger}\right)^{3 / 2}\left(\frac{\partial q^{\star}}{\partial M_{0,3}}\right)^{\dagger}\right]
\end{aligned}
$$

where $C_{2 \mid \alpha}^{\dagger}=C_{2 \mid \alpha} / C_{0,2}=b_{0}+b_{1} \varphi_{\alpha}$,

$$
\begin{gathered}
\left(\frac{\partial C_{2 \mid \alpha}}{\partial M_{0,2}}\right)^{\dagger}=1-\varrho\left(3 \bar{v}^{\dagger}+q_{2}\right) \varphi_{\alpha}, \quad\left(\frac{\partial C_{2 \mid \alpha}}{\partial M_{0,3}}\right)^{\dagger}=\varrho \varphi_{\alpha} \\
\left(\frac{\partial q^{\star}}{\partial M_{0,2}}\right)^{\dagger}=-3\left[\sum_{\alpha=1}^{4} w_{\alpha}\left(C_{2 \mid \alpha}^{\dagger}\right)^{3 / 2}\right]^{-1}\left[\bar{v}^{\dagger}-\varrho^{2}\left(3 \bar{v}^{\dagger}+q_{2}\right)+\frac{1}{2} q^{\star} \sum_{\alpha=1}^{4} w_{\alpha}\left(C_{2 \mid \alpha}^{\dagger}\right)^{1 / 2}\left(\frac{\partial C_{2 \mid \alpha}}{\partial M_{0,2}}\right)^{\dagger}\right]
\end{gathered}
$$

and

$$
\left(\frac{\partial q^{\star}}{\partial M_{0,3}}\right)^{\dagger}=\left[\sum_{\alpha=1}^{4} w_{\alpha}\left(C_{2 \mid \alpha}^{\dagger}\right)^{3 / 2}\right]^{-1}\left[1-3 \varrho^{2}-\frac{3}{2} q^{\star} \sum_{\alpha=1}^{4} w_{\alpha}\left(C_{2 \mid \alpha}^{\dagger}\right)^{1 / 2}\left(\frac{\partial C_{2 \mid \alpha}}{\partial M_{0,3}}\right)^{\dagger}\right]
$$

From Theorem 1, the matrix $J$ is diagonalizable with five distinct eigenvalues $\lambda_{\alpha}=\bar{u}+\sqrt{C_{2,0}} \varphi_{\alpha}$. Matrix $A_{1}$ is also diagonalizable with the following two eigenvalues:

$$
\lambda_{ \pm}=\bar{u}+\frac{1}{2} \sqrt{C_{2,0}}\left(q_{1} \pm \sqrt{4+q_{1}^{2}}\right) .
$$

Neither of these eigenvalues is an eigenvalue of $J$. The eigenvalue

$$
\lambda_{10}=\frac{\partial \bar{M}_{1,4}}{\partial M_{0,4}}=\bar{u}+\sqrt{C_{2,0}} \sum_{\alpha=1}^{4} w_{\alpha} \varphi_{\alpha} C_{2 \mid \alpha}^{2} \frac{\partial \eta^{\star}}{\partial C_{0,4}}=\bar{u}+\sqrt{C_{2,0}} \mathcal{K}_{2},
$$

where

$$
\mathcal{K}_{2}=\frac{\sum_{\alpha=1}^{4} w_{\alpha} \varphi_{\alpha}\left(C_{2 \mid \alpha}^{\dagger}\right)^{2}}{\sum_{\alpha=1}^{4} w_{\alpha}\left(C_{2 \mid \alpha}^{\dagger}\right)^{2}}=\frac{\sum_{\alpha=1}^{4} w_{\alpha} \varphi_{\alpha}\left(b_{0}+b_{1} \varphi_{\alpha}\right)^{2}}{\sum_{\alpha=1}^{4} w_{\alpha}\left(b_{0}+b_{1} \varphi_{\alpha}\right)^{2}}=\frac{b_{1}\left(2 b_{0}+b_{1} q_{1}\right)}{b_{0}^{2}+b_{1}^{2}}
$$

is real and distinct.

It thus remains to show that the two eigenvalues of $A_{2}$ are real and distinct. The two eigenvalues of $A_{2}$ are

$$
\lambda_{ \pm}=\bar{u}-\frac{1}{2} \sqrt{C_{2,0}}\left[\varrho q_{2}-\left(\frac{\partial \bar{C}_{1,3}}{\partial M_{0,3}}\right)^{\dagger} \pm \varrho \sqrt{\Delta}\right]
$$

where $\Delta=\left[\left(a_{11}+a_{22}\right)^{2}-4\left(a_{11} a_{22}-a_{12} a_{21}\right)\right] /\left(\varrho^{2} C_{2,0}\right)$. Thus, a sufficient condition is that $\Delta>0$ where

$$
\Delta=\left[\frac{1}{\varrho}\left(\frac{\partial \bar{C}_{1,3}}{\partial M_{0,3}}\right)^{\dagger}+q_{2}\right]^{2}+\frac{4}{\varrho}\left[3 \bar{v}^{\dagger}\left(\frac{\partial \bar{C}_{1,3}}{\partial M_{0,3}}\right)^{\dagger}+\left(\frac{\partial \bar{C}_{1,3}}{\partial M_{0,2}}\right)^{\dagger}\right] .
$$

It is straightforward to show that $\Delta$ does not depend on $\bar{v}^{\dagger}$ so that it can be evaluated at $\bar{v}^{\dagger}=0$. Expanding $\Delta\left(\varrho, q_{1}, q_{2}, \eta_{1}\right)$ about $\varrho=0$ yields

$$
\Delta=4\left(3+q_{2}^{2}\right)+3 q_{1} q_{2}\left(2+q_{2}^{2}\right) \varrho+\mathcal{O}\left(\varrho^{2}\right),
$$

and thus $\Delta>12$ for small $|\varrho|$. This concludes the proof. 
For larger $|\varrho|$, the condition that $C_{2 \mid \alpha}^{\dagger}=b_{0}+b_{1} \varphi_{\alpha}>0$, where

$$
\begin{aligned}
& \varphi_{1}=\frac{1}{2}\left(q_{1}+\sqrt{4 \eta_{1}-3 q_{1}^{2}+4 \sqrt{\left(\eta_{1}-q_{1}^{2}\right)\left(\eta_{1}-q_{1}^{2}-1\right)}}\right)>0, \\
& \varphi_{4}=\frac{1}{2}\left(q_{1}-\sqrt{4 \eta_{1}-3 q_{1}^{2}+4 \sqrt{\left(\eta_{1}-q_{1}^{2}\right)\left(\eta_{1}-q_{1}^{2}-1\right)}}\right)<0,
\end{aligned}
$$

leads to two functions that must be positive:

$$
\begin{aligned}
& g_{1}\left(\varrho, q_{1}, q_{2}, \eta_{1}\right)=1+\varrho q_{2}\left|\varphi_{1}\right|-\varrho^{2}\left(q_{1}\left|\varphi_{1}\right|+1\right)>0 \\
& g_{4}\left(\varrho, q_{1}, q_{2}, \eta_{1}\right)=1-\varrho q_{2}\left|\varphi_{4}\right|+\varrho^{2}\left(q_{1}\left|\varphi_{4}\right|-1\right)>0
\end{aligned}
$$

For given values of $\left(q_{1}, q_{2}, \eta_{1}\right)$, these functions provide bounds on $\varrho: \varrho_{\min }<\varrho<\varrho_{\max }$. From (54), $\Delta\left(\varrho, q_{1}, q_{2}, \eta_{1}\right)$ can be found explicitly using symbolic software. For $\varrho_{\min }<\varrho<\varrho_{\max }$ it can be shown that $\Delta>12$ when $\eta_{1} \geq 1+q_{1}^{2}$. When a conditional variance $C_{2 \mid 1}=0$ or $C_{2 \mid 4}=0$, it is likely that $\Delta>0$ for all realizable values of $|\varrho| \leq 1$ so the that the moment system in (52) remains at least weakly hyperbolic. The numerical examples in $\S 6.1$ support this conjecture. As noted earlier, when limiters are applied to keep the conditional variances non-negative, some of the second-order and higher central moments are not reproduced.

\subsection{Kinetic-based flux}

The moment transport system (51) has the form

$$
\partial_{t} \mathbf{M}+\partial_{\mathbf{x}} \cdot \mathbf{F}(\mathbf{M})=\overline{\mathbf{A}}
$$

with flux vector $\mathbf{F}=\left(\mathbf{F}_{x}, \mathbf{F}_{y}\right)^{t}$ for the 10-moment vector $\mathbf{M}$. In our numerical implementation (see $\S 3.3$ ), the components of the fluxes for moment $M_{i, j}$ are computed using a kinetic-based definition:

$$
\begin{aligned}
& F_{x ; i, j}=\int_{\mathbb{R}}\left(\int_{0}^{\infty} f(t, \mathrm{x}, \mathrm{v}) u^{i+1} v^{j} \mathrm{~d} u\right) \mathrm{d} v+\int_{\mathbb{R}}\left(\int_{-\infty}^{0} f(t, \mathrm{x}, \mathrm{v}) u^{i+1} v^{j} \mathrm{~d} u\right) \mathrm{d} v, \\
& F_{y ; i, j}=\int_{\mathbb{R}}\left(\int_{0}^{\infty} f(t, \mathrm{x}, \mathrm{v}) u^{i} v^{j+1} \mathrm{~d} v\right) \mathrm{d} u+\int_{\mathbb{R}}\left(\int_{-\infty}^{0} f(t, \mathrm{x}, \mathrm{v}) u^{i} v^{j+1} \mathrm{~d} v\right) \mathrm{d} u .
\end{aligned}
$$

Thus, for the non-degenerate case, we follow the example in (30) and use the flux-based quadrature in (48):

$$
F_{x ; i, j}=M_{0,0} \sum_{\alpha=1}^{4} w_{\alpha}\left[\max \left(0, \lambda_{\alpha}\right)^{i+1}+\min \left(0, \lambda_{\alpha}\right)^{i+1}\right] \Theta_{u, \alpha}^{j}
$$

where the $u$-conditioned $j$ th-order moment of $v$ is defined by

$$
\Theta_{u, \alpha}^{j}:=\sum_{\beta=1}^{3} \rho_{\alpha \beta}\left(\bar{v}+\bar{v}_{\alpha}+v_{\alpha \beta}\right)^{j}=\sum_{\beta=1}^{4} w_{\beta}^{\star}\left(\bar{v}+\bar{v}_{\alpha}+C_{2 \mid \alpha}^{1 / 2} \varphi_{\beta}^{\star}\right)^{j}
$$

with weights $w_{\beta}^{\star}$ and eigenvalues $\varphi_{\beta}^{\star}$ found from $\left(0,1, q^{\star}, \eta^{*}\right)$ as in (32). This yields

$$
\begin{aligned}
& \Theta_{u, \alpha}^{0}=1, \\
& \Theta_{u, \alpha}^{1}=\bar{v}+\bar{v}_{\alpha}, \\
& \Theta_{u, \alpha}^{2}=\left(\bar{v}+\bar{v}_{\alpha}\right)^{2}+C_{2 \mid \alpha}, \\
& \Theta_{u, \alpha}^{3}=\left(\bar{v}+\bar{v}_{\alpha}\right)^{3}+3\left(\bar{v}+\bar{v}_{\alpha}\right) C_{2 \mid \alpha}+q^{\star} C_{2 \mid \alpha}^{3 / 2}, \\
& \Theta_{u, \alpha}^{4}=\left(\bar{v}+\bar{v}_{\alpha}\right)^{4}+6\left(\bar{v}+\bar{v}_{\alpha}\right)^{2} C_{2 \mid \alpha}+4\left(\bar{v}+\bar{v}_{\alpha}\right) q^{\star} C_{2 \mid \alpha}^{3 / 2}+\eta^{\star} C_{2 \mid \alpha}^{2}
\end{aligned}
$$


where $\bar{v}_{\alpha}, \mu_{\alpha}^{2}, q^{\star}$, and $\eta^{\star}$ are found with the flux-based quadrature and a limiter on $b_{1}$ to ensure $b_{0}+b_{1} \varphi_{\alpha} \geq 0$ for $\alpha=1,4$. The eigenvalues $\lambda_{\alpha}$ and weights $w_{\alpha}$ in (57) are the same as in (30) (i.e., they are found using $q_{1}$ and $\left.\eta_{1}\right)$.

Likewise, for the flux in the $y$ direction,

$$
F_{y ; i, j}=M_{0,0} \sum_{\alpha=1}^{4} w_{\alpha}\left[\max \left(0, \lambda_{\alpha}\right)^{i+1}+\min \left(0, \lambda_{\alpha}\right)^{i+1}\right] \Theta_{v, \alpha}^{j}
$$

where the $v$-conditioned $j$ th-order moments of $u$ is defined by

$$
\Theta_{v, \alpha}^{j}:=\sum_{\beta=1}^{3} \rho_{\alpha \beta}\left(\bar{u}+\bar{u}_{\alpha}+u_{\alpha \beta}\right)^{j}=\sum_{\beta=1}^{4} w_{\beta}^{\star}\left(\bar{u}+\bar{u}_{\alpha}+C_{2 \mid \alpha}^{1 / 2} \varphi_{\beta}^{\star}\right)^{j} .
$$

The eigenvalues $\lambda_{\alpha}$ and weights $w_{\alpha}$ in (60) are defined as in (30), but found using $q_{2}$ and $\eta_{2}$. In other words, the parameters in (57) and (58) are found by conditioning on $u$, while those in (60) and (61) by conditioning on $v$.

\section{Numerical examples}

As example applications, we consider a Riemann problem with 1-D velocity phase space, crossing jets in 2-D, and a Taylor-Green vortex problem with a 2-D velocity phase space. For each case, we solve the moment transport equations in (23) and (51), respectively.

\subsection{1-D Riemann problem}

The 1-D Riemann problem is the same as in [9] where it was used to test 5-moment Gaussian-EQMOM and entropy maximization closures against the analytical solution. The objective here is to illustrate the differences between the hyperbolic flux functions in (29) and (30), and a weakly hyperbolic flux found using 2-node QMOM. Nonetheless, the moment predictions with 3-node HyQMOM are closest to those of the 5moment entropy maximization closure in [9]. In particular, the moments exhibit sub-shocks due to the finite number of velocities used to define the kinetic-based fluxes. In [9], it is shown for the entropy maximization closure that by increasing the number of moments (i.e., seven or nine), the sub-shocks can be eliminated. We would therefore expect to see similar behavior with HyQMOM.

The initial conditions for the 1-D Riemann problem are defined on the real line with a step in the mean velocity at $x=0$ :

$$
\bar{u}=\frac{M_{1}}{M_{0}}= \begin{cases}1 & \text { if } x<0, \\ -1 & \text { otherwise. }\end{cases}
$$

For all $x$, the initial density is unity and the VDF is Maxwellian with energy $C_{2}=1 / 3$. The velocity distribution is assumed initially to be in equilibrium $\left(C_{3}=0, C_{4}=3 C_{2}^{2}\right)$. However, the discontinuous nature of the mean particle velocity quickly leads to particle trajectory crossing (PTC) and a strongly nonequilibrium VDF. Although we do not do so here, the HyQMOM closure can be used with $C_{4}>3 C_{2}^{2}$, which is not the case for the 5-moment closures in [9].

In order to solve the moment equations numerically, the 1-D computational domain $-0.5<x<0.5$ is discretized into 4002 finite-volume cells. The spatial fluxes are treated using the first-order kinetic-based approach described in detail in [9]. The time step is chosen based on the largest magnitude of the abscissas $u_{\alpha}$ used to define the spatial fluxes with a CFL number of 0.8. Note that the maximum CFL number is determined from the largest eigenvalue of the spatial flux (e.g., from (26) for 3-node HyQMOM).

Simulation results for the 1-D Riemann problem using HyQMOM are presented in Figs. 1-3 at time $t=0.1$. For the sake of comparison, the analytical solution is also presented along with results found with the weakly hyperbolic 2-node QMOM [6], based on a delta-function reconstruction using the first four moments. We observe from Fig. 1 that the equilibrium condition is still present on the left and right sides of 

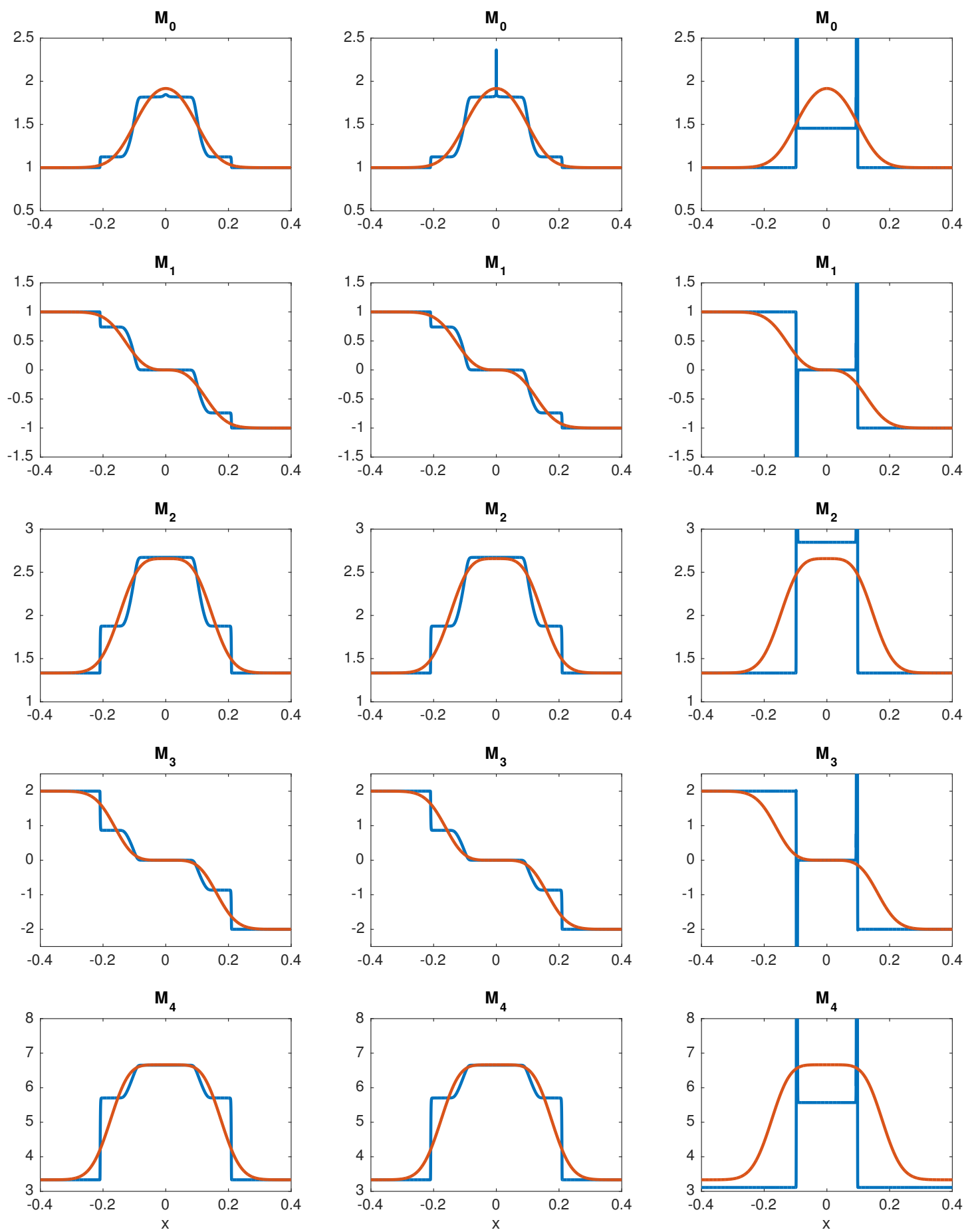

(a) HyQMOM - eigenvalues flux

(b) HyQMOM - 3-node flux

(c) QMOM - 2-node flux

Figure 1: Five moments $\left(M_{0, \ldots, 4}\right)$ in 1-D Riemann problem at $t=0.1$. (a) flux based on four eigenvalues. (b) flux based on 3-node HyQMOM. (c) flux based on 2-node QMOM. Moment method (blue line) versus analytical solution (red line). The moment method in column (c) exhibits $\delta$-shocks. 

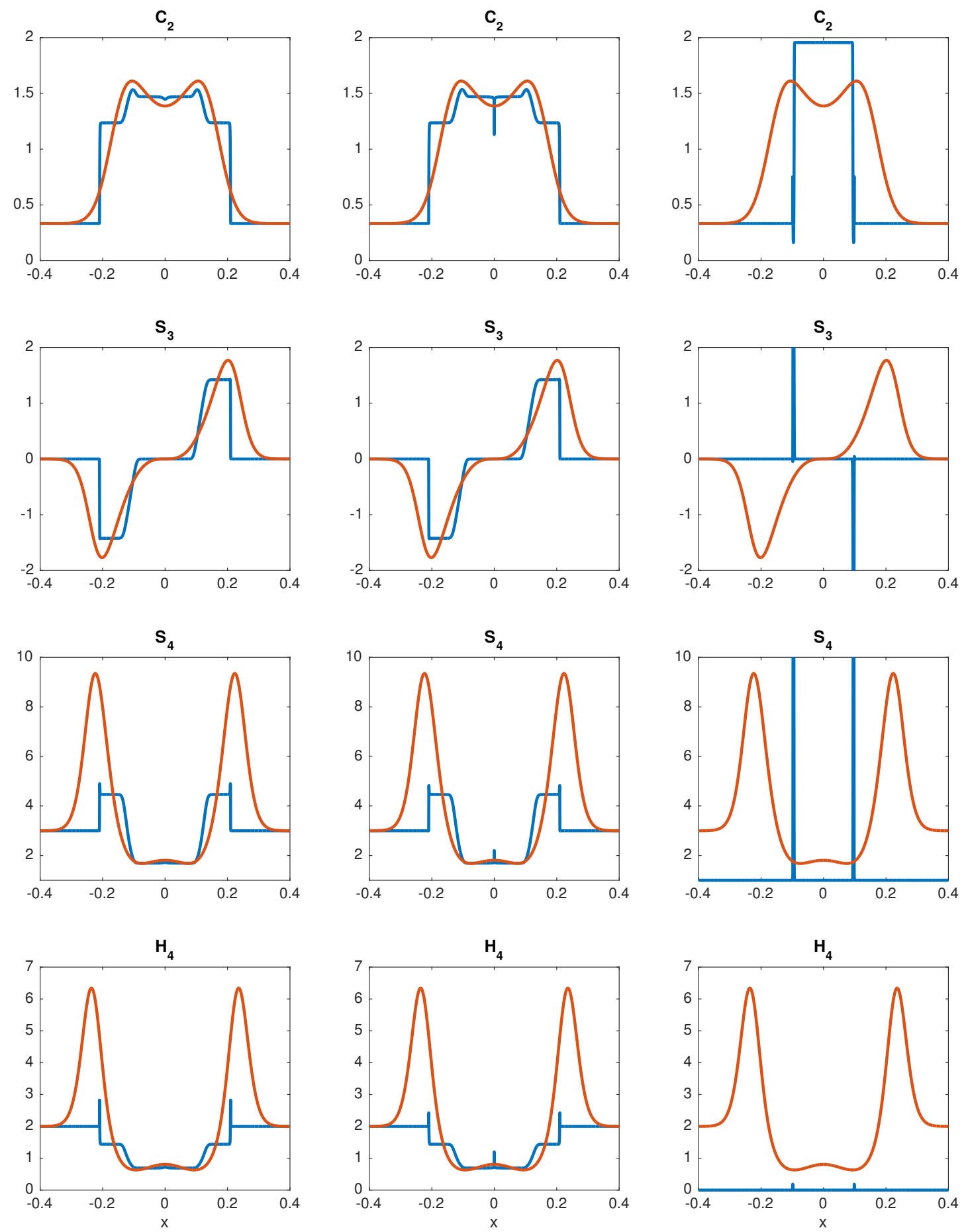

(a) HyQMOM - eigenvalues flux

(b) HyQMOM - 3-node flux

(c) QMOM - 2-node flux

Figure 2: Central moments $\left(C_{2}, S_{3}=q, S_{4}=\eta\right)$ and Hankel determinant $\left|\mathbf{H}_{4}\right|$ for 1-D Riemann problem at $t=0.1$. (a) flux based on four eigenvalues. (b) flux based on 3-node HyQMOM. (c) flux based on 2-node QMOM. Moment method (blue lines) versus analytical solution (red line). By definition, $\left|\mathbf{H}_{4}\right|=0$ for 2-node QMOM. 

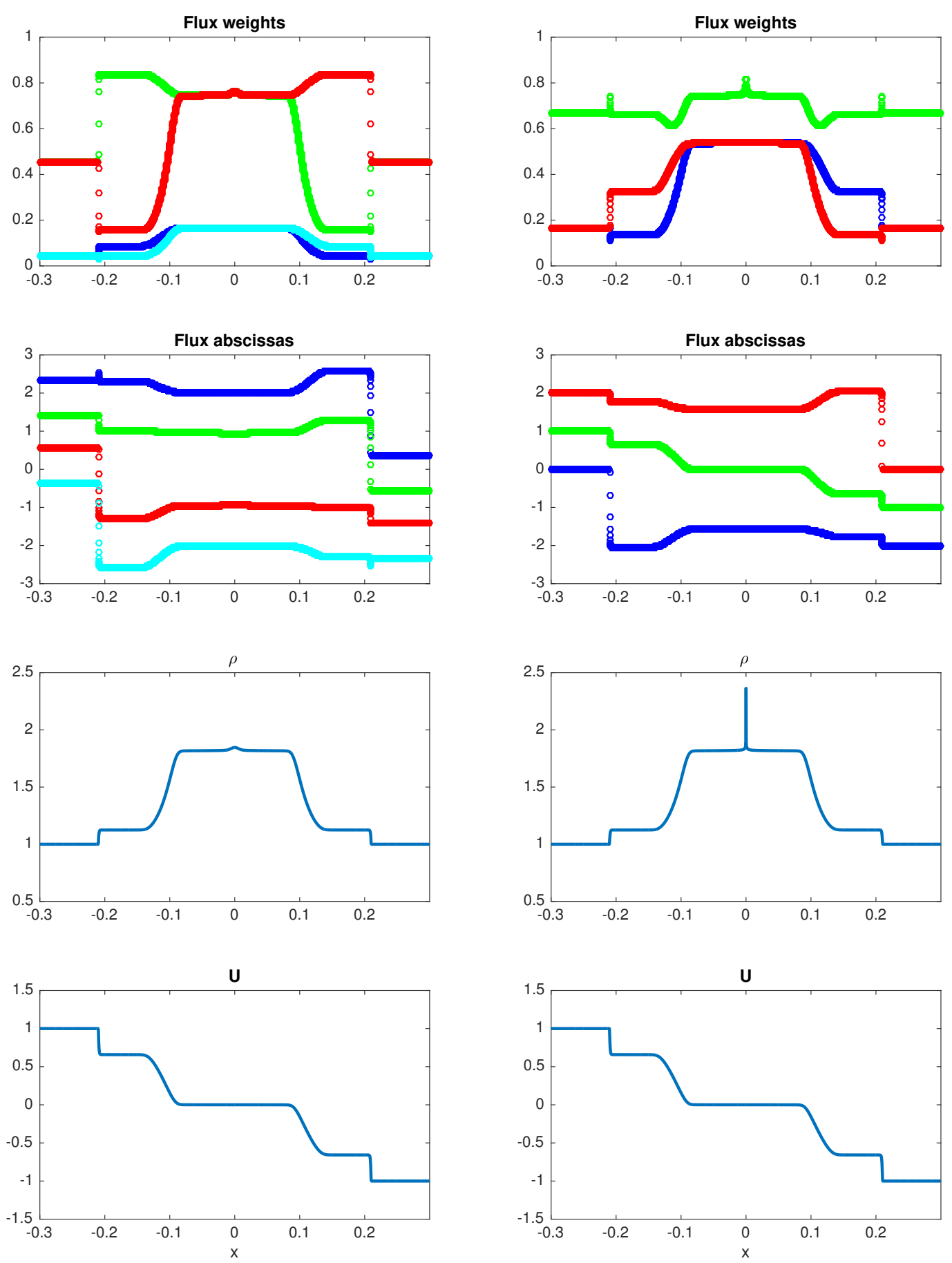

(a) HyQMOM - eigenvalues flux

(b) HyQMOM - 3-node flux

Figure 3: Flux weights and abscissas, and low-order moments $\left(\rho=M_{0}, \bar{u}=M_{1} / M_{0}\right)$ for 1-D Riemann problem at $t=0.1$. (a) flux based on four eigenvalues. (b) flux based on 3-node HyQMOM. Due to the numerical scheme, in the right column the density $\rho$ exhibits a peak at the location where the mean velocity $\bar{u}=0$. 
the computational domain. Note that unlike in a pure PTC problem ${ }^{6}$ where the velocity abscissas remain at their initial values, in Fig. 3 the abscissas have their largest magnitudes just behind the "shock" in density at the edge of the equilibrium domain. This behavior is a direct result of the definition of the spatial fluxes in terms of the underlying 3-node HyQMOM distribution. Indeed, the outer tails of the Gaussian distribution have higher velocity than the value at the peak density and thus penetrate faster into the equilibrium domain, resulting in a higher local flux velocity. The strong deviations from equilibrium are also clearly observed in the central moments in Fig. 2.

Except at the edges of the equilibrium domain, we see from Fig. 1 that when the eigenvalues are used to define the fluxes, the moments are smoothly varying functions of $x$. More importantly, due to the hyperbolicity of the spatial fluxes, the moments are always realizable, and the moment-inversion algorithm always computes a well-defined quadrature from the updated moments. Moreover, the singularities appearing in the solution do not belong to the class of $\delta$-shocks, but to the less singular class of shocks encountered with hyperbolic systems of conservation laws, thus revealing a potentially well-behaved system. Indeed, the peak in $M_{0}$ at $x=0$ observed for the 3-node flux is mainly an artifact due to numerical diffusion classically encountered when using first-order transport schemes [24, 25]. In contrast, when comparing to a weakly hyperbolic method, such as 2-node QMOM in Figs. 1(c) and 2(c), the advantage of guaranteeing hyperbolicity is obvious: QMOM generates stiff discontinuities that are (i) unphysical and (ii) highly constraining in terms of mesh refinement. This type of singularity is also produced using 3-node QMOM (not shown here) for the 6-moment system $\left(M_{0}, M_{1}, \ldots, M_{5}\right)^{t}$.

Overall, the 5-moment closure employed in 3-node HyQMOM yields a robust numerical algorithm. In comparison to QMOM [24], the 3-node HyQMOM provides a higher-fidelity flux representation for a fixed number of moments. Moreover, because the moments of the HyQMOM distribution can be computed to any desired order, the flux representation described in $\S 3.3$ has the potential to be systematically improved. This advantage becomes even more significant for 2-D phase spaces where the number of moments needed for the delta-function reconstruction increases rapidly with the order of the moments [24].

\subsection{2-D crossing jets with a compressive gas field}

As in [26], CHyQMOM is applied here to simulate the crossings of two jets of particles in a compressible carrier phase by solving the 10-moment system in (51). The gaseous flow field is the following:

$$
u_{g}(x, y)=u_{g, 0}, \quad v_{g}(x, y)=\epsilon(y-1)
$$

and the acceleration terms are

$$
\mathcal{A}_{x}=\frac{1}{\tau_{p}}\left(u-u_{g}\right), \quad \mathcal{A}_{y}=\frac{1}{\tau_{p}}\left(v-v_{g}\right)
$$

where $u_{g, 0}=0.2 \mathrm{~m} \mathrm{~s}^{-1}$ is the axial gas velocity and $\epsilon=1 \mathrm{~s}^{-1}$ is the rate of strain in the vertical direction. The dynamics of particles in this flow field are characterized by the Stokes number $\mathrm{St}=\epsilon \tau_{p}$, whose critical value $\mathrm{St}_{c}=1 / 4$ delineates two regimes: for $\mathrm{St}<\mathrm{St}_{c}$, the particles are exponentially relaxing towards the centerline $y=1 \mathrm{~m}$, while for $\mathrm{St}>\mathrm{St}_{c}$ the particles will in addition oscillate around the centerline.

In Fig. 4, particles are injected with zero vertical velocity and the same axial velocity as the gas phase, through two slots at $x=0$ and $y \in[0.4,0.6]$ and $y \in[1.4,1.6]$, and with a Stokes number St $=20$ St $_{c}$. Because of their high Stokes number and the symmetry of the injection about the centerline, the two jets cross each other at the centerline. It can be seen that CHyQMOM properly handles the particle trajectories as well as the crossing events. To further validate the ability of CHyQMOM to reproduce crossing events, the PDF of the vertical velocity component is presented in Fig. 5 at the three crossing locations. For CHyQMOM, these velocities correspond to the flux-based quadrature, i.e., to $\lambda_{\alpha}$. It is worth mentioning that the analytical solution of the proposed configuration does not lead to a bidisperse velocity but to a double-window solution. Here we observe that the predicted velocities are close to the limits of the analytical solution.

${ }^{6} \mathrm{~A}$ pure PTC problem corresponds to the initial condition with $C_{2}=0$, which is on the boundary of moment space. 


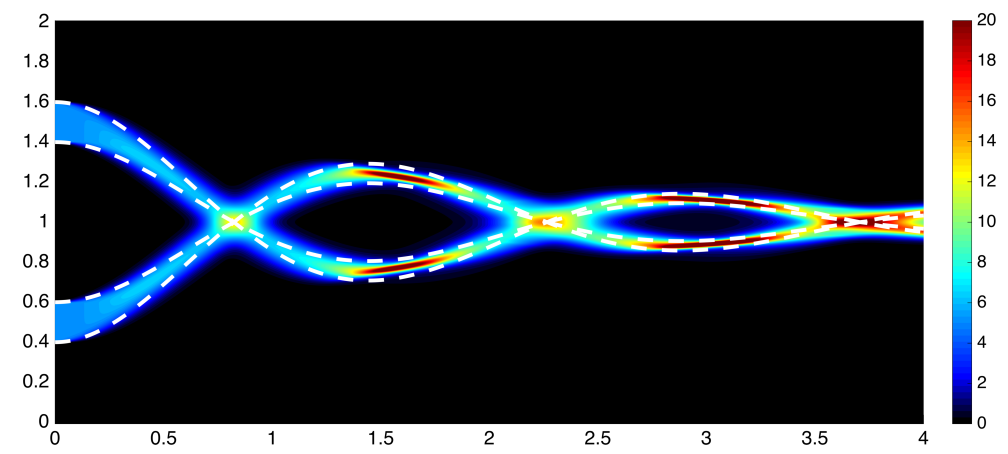

Figure 4: Number density in two-jet crossing with $\mathrm{St}=20 \mathrm{St}_{c}$ at steady state for CHyQMOM. White dashed lines represent the trajectories delineating the region where the particles lie in the analytical solution [26].
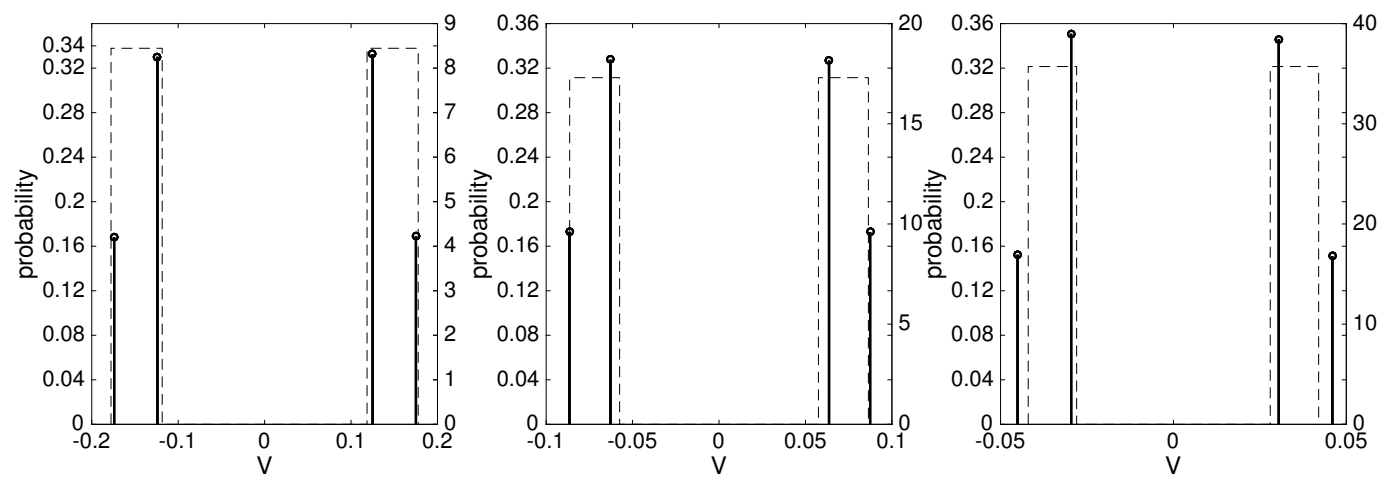

Figure 5: Probability density function for the vertical velocity at the three first crossing locations (from left to right) in two-jet crossing with $\mathrm{St}=20 \mathrm{St}_{c}$ at steady state for CHyQMOM (continuous black lines) and analytical solution (dashed lines). 

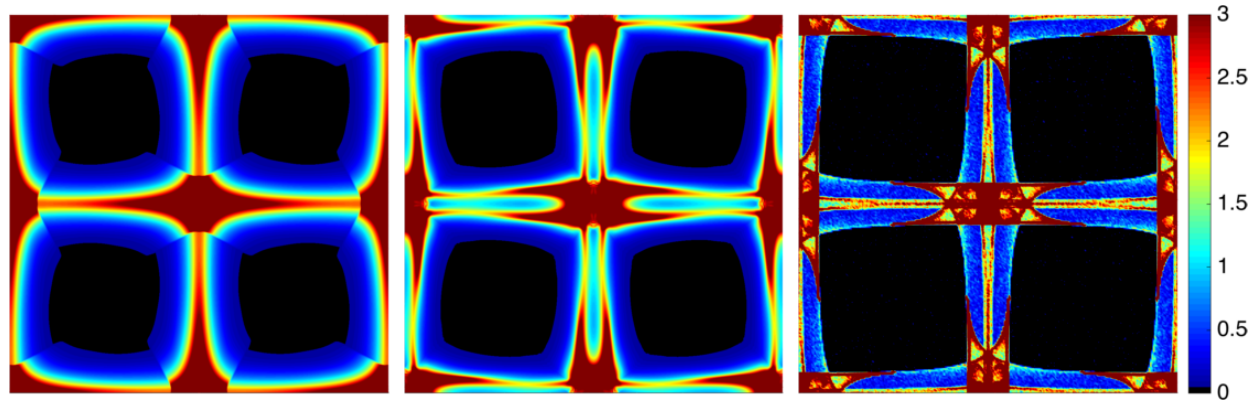

Figure 6: Number density in Taylor-Green vortices with $\mathrm{St}=5 \mathrm{St}_{c}$ at time $t=2$ for AG (left), CHyQMOM (center) and Lagrangian (right) simulations.

\subsection{2-D Taylor-Green vortices}

In this section, CHyQMOM is applied to simulate a 2-D Taylor-Green flow with Stokes drag [26] by solving the 10-moment system in (51). The gas-phase velocity components are

$$
u_{g}(x, y)=\sin (2 \pi x) \cos (2 \pi y), \quad v_{g}(x, y)=-\cos (2 \pi x) \sin (2 \pi y) ;
$$

and the acceleration terms are

$$
\mathcal{A}_{x}=\frac{1}{\mathrm{St}}\left(u-u_{g}\right), \quad \mathcal{A}_{y}=\frac{1}{\mathrm{St}}\left(v-v_{g}\right) .
$$

With these definitions, the moment acceleration terms from (50) are closed. The moment system is solved on a unit-square domain with grid resolutions $256^{2}, 512^{2}$ and $1024^{2}$ to illustrate grid convergence. Unless stated otherwise, all results are shown for the $512^{2}$ resolution. For comparison, a 6-moment system (i.e., up to second order) with the anisotropic Gaussian (AG) closure [26] and a Lagrangian particle method are solved on the same grids. Details concerning the latter can be found in [26] where similar comparisons are made with a 4-moment isotropic Gaussian (IG) closure. At time $t=0$, the particles are uniformly distributed in the computational domain with zero velocity.

For 2-D Taylor-Green flow, there exists a critical Stokes number $\mathrm{St}_{c}=\frac{1}{8 \pi}[26]$, below which the particle velocity variance is null (i.e., the central moments are null) and above which particle trajectory crossings (PTC) occur, making the particle velocity distribution multi-modal and the central moments non-null. When $\mathrm{St}=\mathrm{St}_{c}$, all particles accumulate at the edges of the vortices (see Fig. 9 in [26]). By its nature, the Lagrangian simulation can capture all PTC events and thus yields the highest fidelity solution. In contrast, the AG closure does not allow for PTC (which requires knowledge of the third-order moments), while the CHyQMOM closure can capture (locally) one PTC event. In any case, when the moment closures are unable to reproduce the multi-modal velocity distribution, the number density field $M_{0,0}$ is 'smoothed out' relative to the Lagrangian field.

In Figs. 6-8, number density fields at $t=2$ are shown for the three methods at three different Stokes numbers: $5 \mathrm{St}_{c}, 10 \mathrm{St}_{c}$ and $20 \mathrm{St}_{c}$, respectively. In comparison to $\mathrm{AG}$, the CHyQMOM result in Fig. 6 captures the primary PTC seen in the Lagrangian result for $\mathrm{St}=5 \mathrm{St}_{c}$. However, as expected, near the center of the domain where multiple PTC occur, the moment closures cannot capture the fine details present in the Lagrangian simulation. As the Strokes number increases, more and more PTC occur. For the largest Stokes number shown in Fig. 8, CHyQMOM captures more fine details as compared to AG, but both moment closures are significantly 'smoother' than the Lagrangian field.

As done in [26], the time-dependent behaviors of the three simulation methods are compared using the following statistics:

$$
g_{p}=\frac{\left\{M_{0,0}^{2}\right\}}{\left\{M_{0,0}\right\}^{2}}, \quad \delta \tilde{\theta}_{p}=\frac{\left\{M_{0,0}\left(C_{2,0}+C_{0,2}\right)\right\}}{2\left\{M_{0,0}\right\}}, \quad \tilde{E}_{p}=\frac{\left\{M_{2,0}+M_{0,2}\right\}}{2\left\{M_{0,0}\right\}}
$$



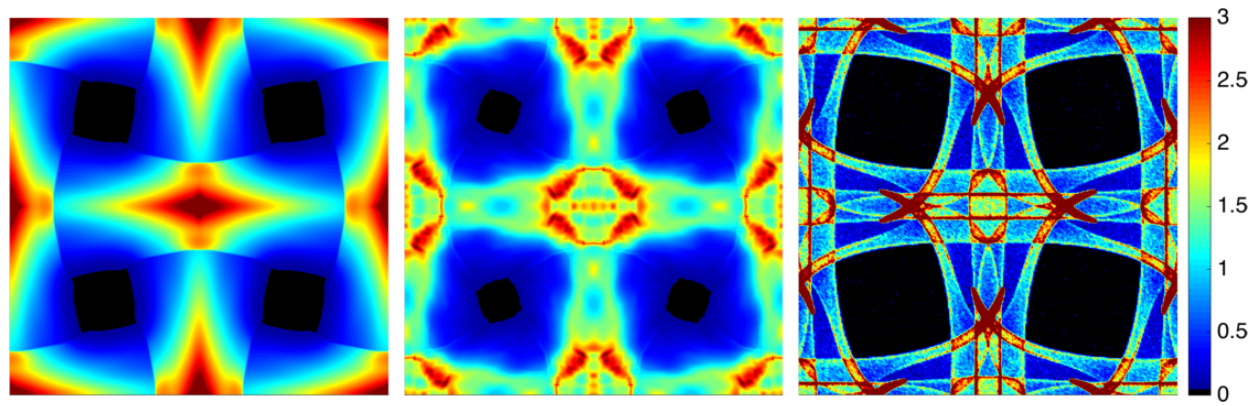

Figure 7: Number density in Taylor-Green vortices with $\mathrm{St}=10 \mathrm{St}_{c}$ at time $t=2$ for AG (left), CHyQMOM (center) and Lagrangian (right) simulations.
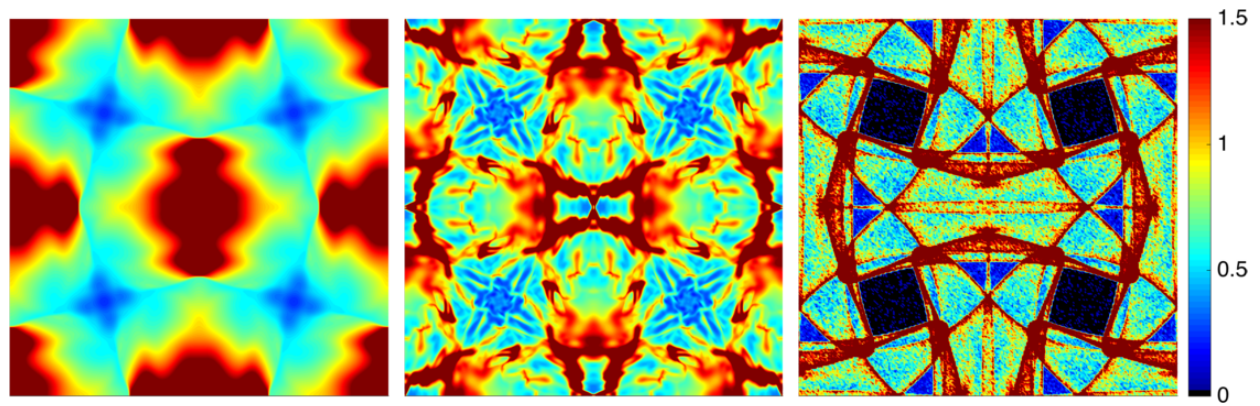

Figure 8: Number density in Taylor-Green vortices with $\mathrm{St}=20 \mathrm{St}_{c}$ at time $t=2$ for AG (left), CHyQMOM (center) and Lagrangian (right) simulations.
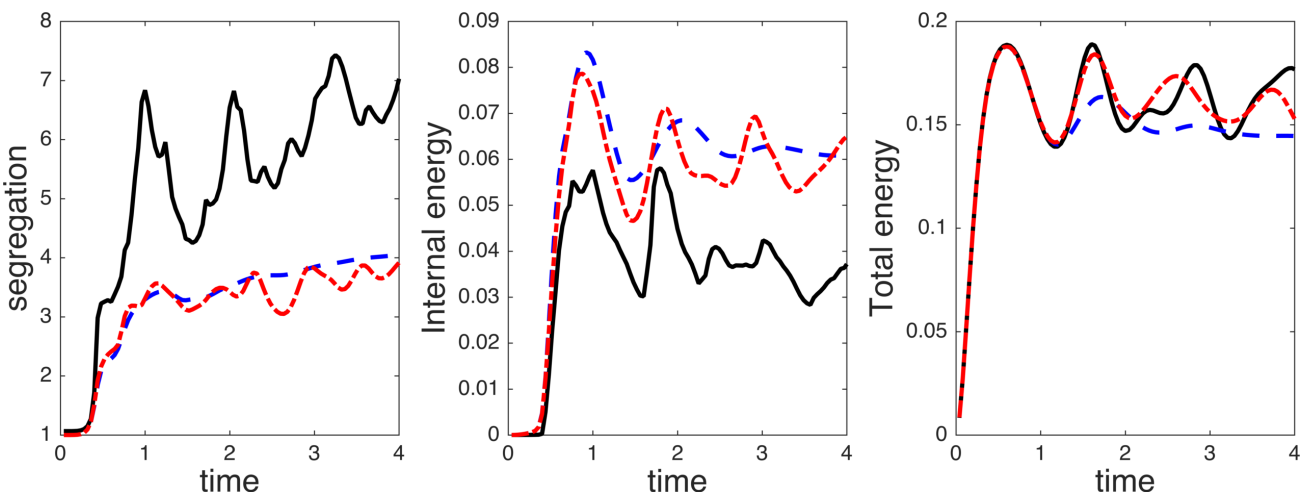

Figure 9: Time evolution of segregation, internal energy and total energy in Taylor-Green vortices with $\mathrm{St}=5 \mathrm{St}_{c}$ for AG $(\mathrm{blue}$ dashed line), CHyQMOM (red dot-dashed line) and Lagrangian (black line) simulations. 

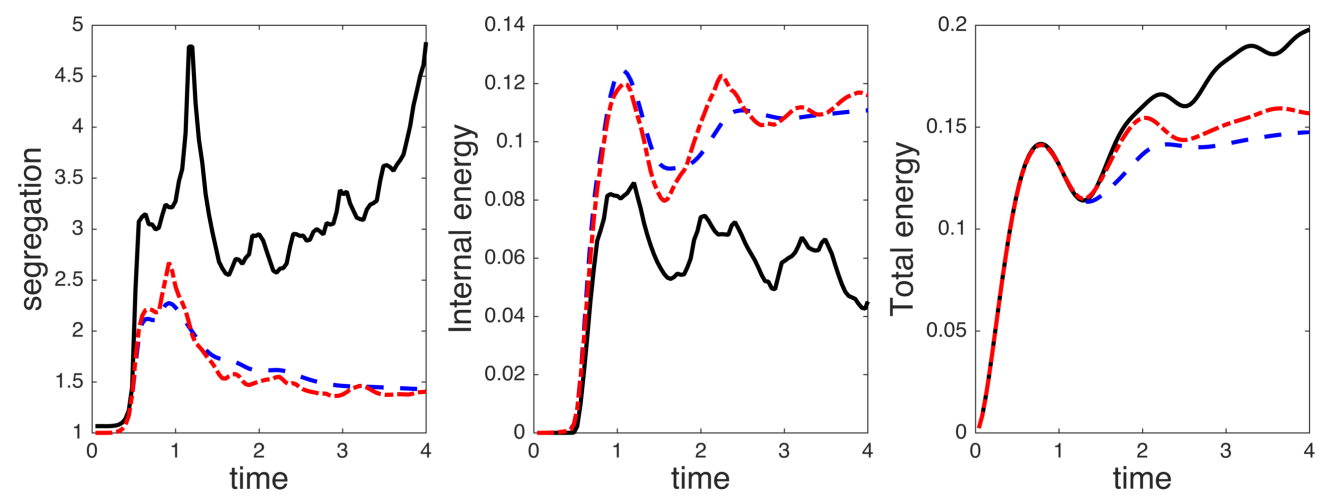

Figure 10: Time evolution of segregation, internal energy and total energy in Taylor-Green vortices with $\mathrm{St}=10 \mathrm{St}_{c}$ for $\mathrm{AG}$ (blue dashed line), CHyQMOM (red dot-dashed line) and Lagrangian (black line) simulations.
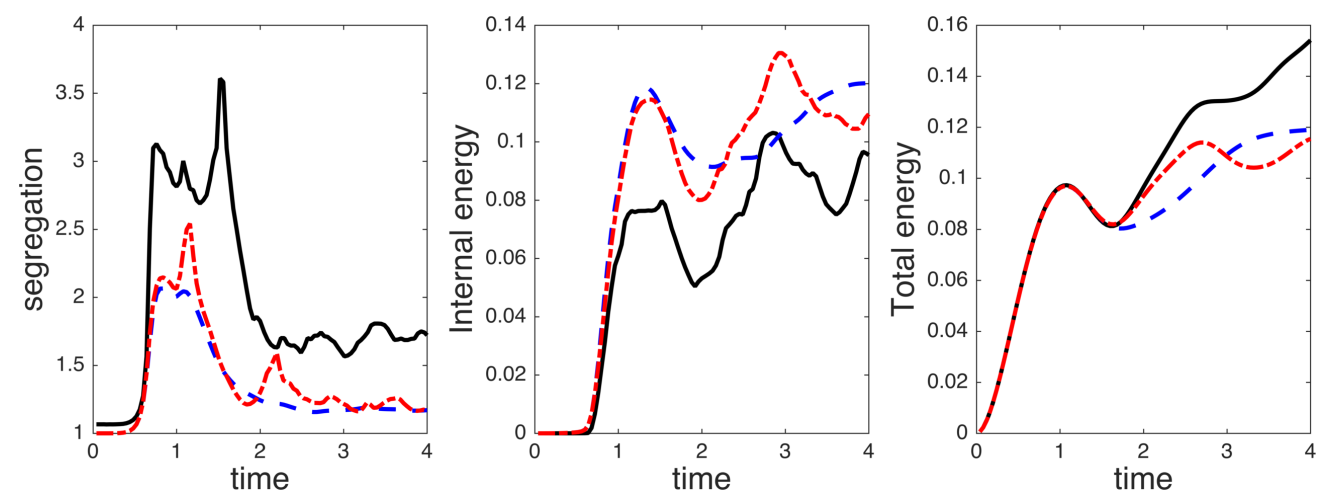

Figure 11: Time evolution of segregation, internal energy and total energy in Taylor-Green vortices with $\mathrm{St}=20 \mathrm{St} \mathrm{t}_{c}$ for $\mathrm{AG}$ (blue dashed line), CHyQMOM (red dot-dashed line) and Lagrangian (black line) simulations. 

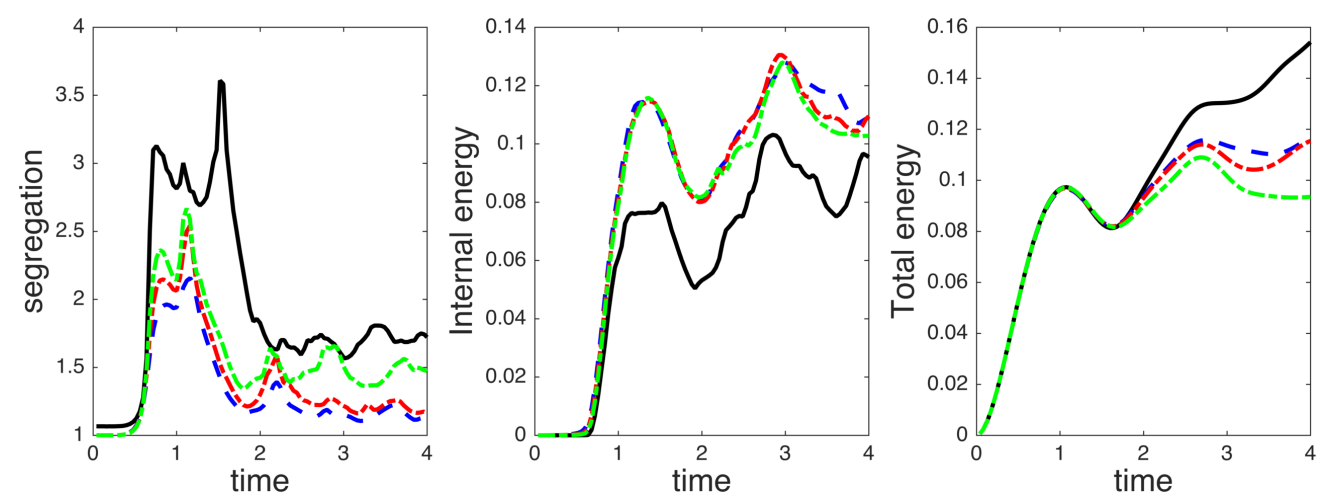

Figure 12: Time evolution of segregation, internal energy and total energy in Taylor-Green vortices with $\mathrm{St}=20 \mathrm{St} \mathrm{c}_{c}$ for Lagrangian (black line) and CHyQMOM with $256^{2}$ (blue), $512^{2}$ (red) and $1024^{2}$ (green) mesh cells.

where $\{\cdot\}$ denotes the spatial average over all grid cells. The segregation index $g_{p}$ measures the degree of non-uniformity of the number density field. The internal energy $\delta \tilde{\theta}_{p}$ is a measure of the level of velocity fluctuations (i.e., the granular temperature or spatially uncorrelated kinetic energy $[27,28,29]$ ), while the total energy $\tilde{E}_{p}$ measure the total kinetic energy transferred from the fluid to the particle phase.

In Figs. 9-11, these statistics are plotted for each simulation method for the three Stokes numbers, respectively. Qualitatively, the statistics for the AG and CHyQMOM closures are very similar (especially when compared to IG statistics in [26]). This would imply that adding more moments by increasing $N$ in CHyQMOM will lead to only a small improvement in the energy statistics as compared to the Lagrangian simulations. Overall, CHyQMOM does a better job than AG in capturing the time-dependence of the statistics, particularly for $t>2$. However, the most obvious advantage of CHyQMOM with $N=3$ over AG is its ability to capture PTC, and thus to provide a higher fidelity representation of the number density field in particle-laden flows with strong vorticity.

In Fig. 12, the dependence of the CHyQMOM statistics on the computational grid is shown for $\mathrm{St}=20 \mathrm{St}_{c}$. In general, for $t<2.5$ the dependence on the grid is small. However, for larger times the segregation is higher for the finer grid. In contrast, the energy statistics are only weakly dependent on the grid resolution. We should emphasize that unlike with CQMOM [4], which is weakly hyperbolic [6], the hyperbolic nature of CHyQMOM should allow for grid-independent solutions on sufficiently fine grids with minimal additional computational cost. Indeed, with the modified CQMOM employed in CHyQMOM, less moments are required with CHyQMOM as compared to the original 2-D CQMOM formulation in [4].

\section{Conclusions}

The conditional hyperbolic quadrature method of moments and the related moment-inversion algorithms introduced in this work appear to be a very promising approach for the direct-numerical simulation of the kinetic equation describing particle-laden turbulent flows [30,31]. The proposed approach combines numerical stability and a lower level of singularity compared to existing quadrature-based moment methods, see [6], and is able to capture both particle trajectory crossing (PTC) caused by the free-transport term and the effects of vortices. It is noteworthy that CHyQMOM naturally degenerates toward the correct velocity distribution with the associated spatial fluxes in both the PTC and dispersion limits. Moreover, by relying on the recent advances in CQMOM [4], the CHyQMOM naturally adapts to the required number of nodes in even highly degenerate cases (e.g., in the absence of particles). As such, the Eulerian moment methods described in this work should offer an attractive alternative to Lagrangian particle tracking methods for simulating particle-laden flows. Here, we have focused on HyQMOM with $N=2$ and 3 nodes, but future work is warranted to determine the constraint on $C_{2 N-1}$ in HyQMOM needed to make the 1-D moment system hyperbolic for $N \geq 4$. 


\section{Acknowledgments}

ROF was partially supported by grants from the U.S. National Science Foundation (CBET-1437865 and ACI-1440443).

\section{Appendix A. Hankel matrices, moment space, and moment constraints}

Let $\mathbf{M}$ be the vector of moments of a $\operatorname{VDF} f(u)$ defined for $u \in \mathbb{R}$. If $0<M_{0}$, let $C_{j}$ be the corresponding central moment of order $j$. The Hankel matrix $\mathbf{H}_{2 n}[14,32]$, defined by

$$
\mathbf{H}_{2 n}=\left[\begin{array}{cccc}
C_{0} & C_{1} & \ldots & C_{n} \\
C_{1} & C_{2} & \ldots & C_{n+1} \\
\vdots & \vdots & \ddots & \vdots \\
C_{n} & C_{n+1} & \ldots & C_{2 n}
\end{array}\right],
$$

is non-negative if $\left|\mathbf{H}_{2 n}\right| \geq 0$. The moments $\mathbf{M}$ up to order $2 n$ are realizable if $\left|\mathbf{H}_{2 m}\right|$ is non-negative for all $m \in\{0,1, \ldots, n\}$. The moments live in the interior of moment space if $\left|\mathbf{H}_{2 m}\right|>0$ for all $m \in\{0,1, \ldots, n\}$, and reside on the boundary if $\left|\mathbf{H}_{2 m}\right|=0$ for some $m \in\{0,1, \ldots, n\}$. Note that if $\left|\mathbf{H}_{2 j}\right|=0$ then $\left|\mathbf{H}_{2 m}\right|=0$ for $m>j$. In the main text, we make use of the conditions $\left|\mathbf{H}_{2}\right|=C_{2} \geq 0$ and $\left|\mathbf{H}_{4}\right|=C_{2} C_{4}-C_{2}^{3}-C_{3}^{2} \geq 0$ as the realizability conditions for the even-order moments for 2-node and 3-node HyQMOM, respectively. For infinite domains, the odd-order moments can take any value in $\mathbb{R}$.

As discussed in the main text, the choice of $C_{2 N-1}$ in HyQMOM is related to the moment fluxes. In order for the Jacobian matrix of the 1-D moment fluxes for the moment system $\mathbf{M}=\left(M_{0}, \ldots, M_{2 N-2}\right)^{t}$ to have an eigenvalue at $\bar{u}$, the following condition must hold for $N \geq 3$ :

$$
\left.\frac{\partial \bar{M}_{2 N-1}}{\partial M_{0}}\right|_{M_{1}=0}=0 \quad \Longrightarrow \quad 2 N=3+\sum_{n=1}^{2 N-4} n \frac{\partial \ln S_{2 N-1}}{\partial \ln S_{n+2}}
$$

where $S_{n}=C_{n} / C_{2}^{n / 2}$ (e.g., $S_{3}=q$ and $\left.S_{4}=\eta\right)$ and $S_{2 N-1}$ depends only on $\left(S_{3}, \ldots, S_{2 N-2}\right)$. For example, with $N=4$ the choice

$$
\begin{aligned}
S_{7}=3 S_{3} S_{6}-S_{4} S_{5}+2 S_{3} S_{4}^{2}-3 S_{3}^{2} S_{5}+a \frac{3}{4}\left(4 S_{4}-\right. & \left.3 S_{3}^{2}\right)\left(S_{3}^{3}-2 S_{3} S_{4}+S_{5}\right) \\
& +b \frac{3\left(10 S_{4}^{2}+6 S_{6}-15 S_{3} S_{5}\right)\left(S_{3}^{3}-2 S_{3} S_{4}+S_{5}\right)}{4\left(4 S_{4}-3 S_{3}^{2}\right)}
\end{aligned}
$$

satisfies (A.2) for all real values of $a$ and $b$. Thus, this choice will result in a normalized eigenvalue at $\varphi_{0}=0$.

From the moment system $\mathbf{M}=\left(M_{0}, \ldots, M_{6}\right)^{t}$, when $\left|\mathbf{H}_{4}\right|=0$ (i.e. $\left.S_{4}=1+S_{3}^{2}\right)$, the choice in (A.3) results in two distinct normalized eigenvalues at \pm 1 , which are triple roots, for all values of $a$ and $b$. For $\left|\mathbf{H}_{6}\right|=0$, (A.3) results in three distinct normalized eigenvalues at $\left(0, \pm \sqrt{S_{4}}\right)$ for $a=1-b$. Note that the eigenvalues for these two cases exactly correspond the QMOM abscissas for $N=2$ and 3, respectively. Numerical tests reveal that $b=2$ and $a=-1$ yields real normalized eigenvalues for $\left|\mathbf{H}_{6}\right|>0$ when $S_{3}=S_{5}=0$ (i.e., for any symmetric VDF). However, it remains to be shown whether (A.3) yields real-valued eigenvalues for all of moment space (e.g., with any highly asymmetric VDF).

\section{Appendix B. 2-D, 9-node CHyQMOM with twelve moments}

In this appendix, we briefly describe the extension of (37) using the symmetrical 12-moment set

$$
\mathbf{M}=\left[\begin{array}{lllll}
M_{0,0} & M_{0,1} & M_{0,2} & M_{0,3} & M_{0,4} \\
M_{1,0} & M_{1,1} & M_{1,2} & & \\
M_{2,0} & M_{2,1} & & & \\
M_{3,0} & & & & \\
M_{4,0} & & & &
\end{array}\right]
$$


for the non-degenerate case. This moment set is of interest because all third-order moments (which control the energy flux) are included. At the same time, the degenerate cases with exactly one or two velocity abscissas needed to handle particle-trajectory crossing at arbitrary angles are allowed. As done in the main text, we assume that $0<M_{0,0}$ and define the reconstruction algorithm using the central moments $C_{i, j}$.

For the central moments found from (B.1), the conditional velocity is written as a second-order polynomial in $u_{\alpha}$, i.e., $\bar{v}_{\alpha}=a_{0}+a_{1} u_{\alpha}+a_{2} u_{\alpha}^{2}$. The coefficients $a_{0}, a_{1}$ and $a_{2}$ are defined by the following constraints:

$$
\sum_{\alpha=1}^{3} \rho_{\alpha} \bar{v}_{\alpha}=C_{0,1}=0, \quad \sum_{\alpha=1}^{3} \rho_{\alpha} u_{\alpha} \bar{v}_{\alpha}=C_{1,1}, \quad \sum_{\alpha=1}^{3} \rho_{\alpha} u_{\alpha}^{2} \bar{v}_{\alpha}=C_{2,1}
$$

which yields a linear system for the coefficients:

$$
\left[\begin{array}{ccc}
1 & 0 & C_{2,0} \\
0 & C_{2,0} & C_{3,0} \\
C_{2,0} & C_{3,0} & C_{4,0}
\end{array}\right]\left[\begin{array}{l}
a_{0} \\
a_{1} \\
a_{2}
\end{array}\right]=\left[\begin{array}{c}
0 \\
C_{1,1} \\
C_{2,1}
\end{array}\right]
$$

This linear system has a unique solution if the central moments $C_{i, 0}$ are in the interior of moment space (i.e., the system is non-degenerate: $C_{2,0}>0$ and $C_{2,0} C_{4,0}>C_{2,0}^{3}+C_{3,0}^{2}$ ). In the degenerate case $C_{2,0}=0$, $\bar{v}_{\alpha}=0$; and when $C_{2,0}>0$ but $C_{2,0} C_{4,0}=C_{2,0}^{3}+C_{3,0}^{2}, \bar{v}_{\alpha}=\frac{C_{1,1}}{C_{2,0}} u_{\alpha} \cdot{ }^{7}$ Note that these two cases correspond to $\rho_{2}=1$ and $\rho_{2}=0$, respectively.

As in the main text, the additional parameters $\left\{\rho_{\alpha 1}, \rho_{\alpha 2}, \rho_{\alpha 3}, v_{\alpha 1}, v_{\alpha 2},\right\}$ are determined from the conditional moments $\left\{1,0, C_{2 \mid \alpha}, C_{3 \mid \alpha}, C_{4 \mid \alpha}\right\}$ using the 3-node HyQMOM in $\S 2.4$. Thus, we compute the coefficients $b_{0}$ and $b_{1}$ in $C_{2 \mid \alpha}=b_{0}+b_{1} u_{\alpha}$ from (41) using $\left\{C_{0,2}, C_{1,2}\right\}$. This yields

$$
\begin{gathered}
\sum_{\alpha=1}^{3} \rho_{\alpha} C_{2 \mid \alpha}=b_{0}=C_{0,2}-\sum_{\alpha=1}^{3} \rho_{\alpha} \bar{v}_{\alpha}^{2}, \\
\sum_{\alpha=1}^{3} \rho_{\alpha} u_{\alpha} C_{2 \mid \alpha}=b_{1} C_{2,0}=C_{1,2}-\sum_{\alpha=1}^{3} \rho_{\alpha} u_{\alpha} \bar{v}_{\alpha}^{2} .
\end{gathered}
$$

If one of the conditional variances is null, then $b_{1}$ is limited such that all conditional variances are nonnegative

As in the main text, the conditional moments $C_{3 \mid \alpha}$ and $C_{4 \mid \alpha}$ are found from $\left\{C_{0,3}, C_{0,4}\right\}$ by assuming that they are depend on $\alpha$ through $C_{2 \mid \alpha}$, i.e., $C_{3 \mid \alpha}=q^{\star} C_{2 \mid \alpha}^{3 / 2}$ and $C_{4 \mid \alpha}=\eta^{\star} C_{2 \mid \alpha}^{2}$. This yields

$$
\begin{gathered}
q^{\star} \sum_{\alpha=1}^{3} \rho_{\alpha} C_{2 \mid \alpha}^{3 / 2}=C_{0,3}-\sum_{\alpha=1}^{3} \rho_{\alpha} \bar{v}_{\alpha}^{3}-3 \sum_{\alpha=1}^{3} \rho_{\alpha} \bar{v}_{\alpha} C_{2 \mid \alpha}, \\
\eta^{\star} \sum_{\alpha=1}^{3} \rho_{\alpha} C_{2 \mid \alpha}^{2}=C_{0,4}-\sum_{\alpha=1}^{3} \rho_{\alpha} \bar{v}_{\alpha}^{4}-6 \sum_{\alpha=1}^{3} \rho_{\alpha} \bar{v}_{\alpha}^{2} C_{2 \mid \alpha}-4 q^{\star} \sum_{\alpha=1}^{3} \rho_{\alpha} \bar{v}_{\alpha} C_{2 \mid \alpha}^{3 / 2},
\end{gathered}
$$

which are solved for $q^{\star}$ and $\eta^{\star}$. The realizability of $C_{4 \mid \alpha}$ requires that $\eta^{\star} \geq 1+\left(q^{\star}\right)^{2}$. If this condition is not met, then $q^{\star}$ and $\eta^{\star}$ are projected to the realizability curve $\eta^{\star}=1+\left(q^{\star}\right)^{2}$ in the direction of the Gaussian moments (i.e., $q^{\star}=0$ and $\eta^{\star}=3$ ). Three-node HyQMOM can then be applied for each $\alpha$ to find the remaining parameters.

\section{Bibliography}

[1] O. Desjardins, R. O. Fox, P. Villedieu, A quadrature-based moment method for dilute fluid-particle flows, J. Comput. Phys. 227 (4) (2008) 2514-2539.

\footnotetext{
${ }^{7}$ In the main text, $\bar{v}_{\alpha}=a_{1} u_{\alpha}$ is used for all of moment space. Here we see that this choice is exact at the boundary of moment space when $\left|\mathbf{H}_{4}\right|=0$.
} 
[2] R. O. Fox, A quadrature-based third-order moment method for dilute gas-particle flow, J. Comput. Phys. 227 (12) (2008) 6313-6350.

[3] R. O. Fox, Higher-order quadrature-based moment methods for kinetic equations, J. Comput. Phys. 228 (2009) $7771-7791$.

[4] C. Yuan, R. O. Fox, Conditional quadrature method of moments for kinetic equations, J. Comput. Phys. 230 (22) (2011) $8216-8246$.

[5] F. Bouchut, S. Jin, X. Li, Numerical approximations of pressureless and isothermal gas dynamics, SIAM J. Numer. Anal. 41 (1) (2003) 135-158.

[6] C. Chalons, D. Kah, M. Massot, Beyond pressureless gas dynamics: quadrature-based velocity moment models, Commun. Math. Sci. 10 (4) (2012) 1241-1272.

[7] V. Vikas, Z. J. Wang, A. Passalacqua, R. O. Fox, Realizable high-order finite-volume schemes for quadrature-based moment methods, J. Comput. Phys. 230 (13) (2011) 5328-5352.

[8] C. Chalons, R. O. Fox, M. Massot, A multi-Gaussian quadrature method of moments for gas-particle flows in a LES framework, in: Proceedings of the Summer Program 2010, Center for Turbulence Research, Stanford University, Stanford, USA, 2010, pp. 347-358.

[9] C. Chalons, R. O. Fox, F. Laurent, M. Massot, A. Vié, Multivariate Gaussian extended quadrature method of moments for turbulent disperse multiphase flow, Multiscale Model. Simul. 15 (4) (2017) 1553-1583.

[10] M. Junk, Domain of definition of Levermore's five-moment system, J. Stat. Phys. 93 (5/6) (1998) $1143-1167$.

[11] C. D. Levermore, W. J. Morokoff, The Gaussian moment closure for gas dynamics, SIAM J. Appl. Math. 59 (1996) $72-96$.

[12] C. D. Levermore, Moment closure hierarchies for kinetic theories, J. Stat. Phys. 83 (1996) $1021-1065$.

[13] C. Yuan, F. Laurent, R. O. Fox, An extended quadrature method of moments for population balance equations, J. Aerosol Sci. 51 (2012) 1-23.

[14] W. Gautschi, Orthogonal Polynomials: Computation and Approximation, Oxford University Press, Oxford, UK, 2004.

[15] S. Deshpande, A second order accurate, kinetic theory based, method for inviscid compressible flows, Technical Report 2613, NASA Langley (1986).

[16] B. Perthame, Kinetic formulation of conservation laws, Vol. 21 of Oxford Lecture Series in Mathematics and its Applications, Oxford University Press, Oxford, UK, 2002.

[17] D. L. Wright, Numerical advection of moments of the particule size distribution in Eulerian models, J. Aerosol Sci. 38 (3) (2007) 352-369.

[18] F.-H. Vasilescu, Hamburger and Stieltjes moment problems in several variables, Transactions of the American Mathematical Society 354 (3) (2002) 1265-1278. doi:10.1090/S0002-9947-01-02943-9. URL http://www . ams . org/tran/2002-354-03/S0002-9947-01-02943-9/

[19] L. Fialkow, S. Petrovic, A moment matrix approach to multivariate cubature, Integr. Equ. Oper. Theory 52 (1) (2005) $85-124$.

[20] C. Kleiber, J. Stoyanov, Multivariate distributions and the moment problem, J. Multivariate Analysis 113 (Supplement C) (2013) 7-18. doi:10.1016/j.jmva.2011.06.001.

URL http://www.sciencedirect.com/science/article/pii/S0047259X11001138

[21] L. Qi, Hankel tensors: Associated Hankel matrices and Vandermonde decomposition, arXiv:1310.5470 [math] ArXiv: 1310.5470 .

URL http://arxiv.org/abs/1310.5470

[22] H. Dette, D. Tomecki, Hankel determinants of random moment sequences, arXiv:1508.00617 [math]ArXiv: 1508.00617. URL http://arxiv.org/abs/1508.00617

[23] L. Qi, A note on the multidimensional moment problem, arXiv:1602.03406 [math]ArXiv: 1602.03406. URL http://arxiv.org/abs/1602.03406

[24] R. O. Fox, Optimal moment sets for multivariate direct quadrature methods of moments, Indust. \& Engng. Chem. Res. 48 (2009) 9686-9696.

[25] D. Kah, A. Vié, C. Chalons, M. Massot, Second-order scheme for quadrature-based velocity high order moment methods for disperse two-phase flows, Annu. Res, Brief CTR, (2011) 321-334.

[26] A. Vié, F. Doisneau, M. Massot, On the anisotropic Gaussian velocity closure for inertial-particle laden flows, Commun. Comput. Phys. 17 (01) (2015) 1-46.

[27] P. Février, O. Simonin, K. D. Squires, Partitioning of particle velocities in gas-solid turbulent flow into a continuous field and a spatially uncorrelated random distribution: theoretical formalism and numerical study, J. Fluid Mech. 533 (2005) $1-46$.

[28] R. O. Fox, On multiphase turbulence models for collisional fluid-particle flows, J. Fluid Mech. 742 (2014) $368-424$.

[29] J. Capecelatro, O. Desjardins, R. O. Fox, On fluid-particle dynamics in fully developed cluster-induced turbulence, J. Fluid Mech. 780 (2015) 578-635.

[30] S. Balachandar, J. K. Eaton, Turbulent dispersed multiphase flow, Annu. Rev. Fluid Mech. 42 (2010) $111-133$.

[31] R. O. Fox, Large-eddy-simulation tools for multiphase flows, Annu. Rev. Fluid Mech. 44 (2012) 47-76.

[32] H. Dette, W. J. Studden, The Theory of Canonical Moments with Applications in Statistics, Probability, and Analysis, Wiley Series in Probability and Statistics: Applied Probability and Statistics, John Wiley \& Sons Inc., New York, 1997. 